\title{
Fostering Dialogue in the Calculus Classroom Using Dynamic Digital Technology
}

\author{
Patricia Salinas $^{1}$ (D) Eliud Quintero ${ }^{1}$. \\ Juan Manuel Fernández-Cárdenas ${ }^{1}$
}

Published online: 18 February 2016

C) Springer International Publishing 2016

\begin{abstract}
In this article, we discuss the dynamic digital software SimCalc MathWorlds and its potential to promote dialogue in the first calculus course for engineering students at Tecnológico de Monterrey, México. Sixty students participated in a pedagogical sequence of tasks that had been designed to help them appropriate the relations between a function and its derivative. In the classroom, the software provided a visual scenario supporting the various tasks. The simulation of cartoon motion over a straight line was included during the interaction. Corresponding graphs of position and velocity gave meaning to the function and its derivative. Active and exploratory visual perception allowed the interpretation of mathematical relations being sought as affordances provided by the software. Co-action between students and mathematical knowledge through software use promoted dialogue in order to identify those relations as invariants. A qualitative method, predominantly ethnographic, was applied during the 2 weeks of the classroom experience. The results revealed the students' appropriation of the relations by means of mathematical language. With this experience, we propose the term 'dialogic ecosystem' as a way to emphasize the design and performance of the pedagogical sequence, where the teacher, students and software cohabit in an environment resulting in dialogue as an important component for the acquisition of mathematical knowledge.
\end{abstract}

Keywords Calculus $\cdot$ Dialogue $\cdot$ Dialogic ecosystem $\cdot$ Co-action $\cdot$ SimCalc $\cdot$ Digital technology

Patricia Salinas

npsalinas@itesm.mx

1 Tecnológico de Monterrey, Monterrey, Mexico 


\section{Introduction}

According to Sinclair and Robutti (2014), teaching practices in digital environments have mainly related to supporting the organization of teachers' work. But during the last decade or so, the evolution of technology has also supported new ways of doing and representing mathematics. Developments have resulted in an integrated system for communicative and representational expressivity, allowing significant access to graphical representations of mathematical content. We see in these visual elements an opportunity to change the way we 'talk' about and learn mathematics.

Since 2000, we have been working as teachers, researchers and curriculum developers in our institution. We too have encountered the local constraints and broader policy environments that Vahey et al. (2013) claim are important issues in order to advance understanding in our field. In this article, we illustrate some advantages that dynamic mathematical technology offers the learning of mathematics, in particular the calculus curriculum. A central goal concerning our work is the promotion of students' interaction with digital technology in support of a visual and dynamic perception of mathematics.

In addition to the use of novel technology, however, we have been promoting a redesign of the calculus syllabus, supported by our research agenda, and drawing on several graduate theses related to the historical genesis of calculus, aiming to rescue the historical meaning of calculus before adjusting it for students in our classrooms, with a strong component of technology. This meaning is related to an epistemology of calculus content. These are the narratives we look to foster in students, by talking about a real problem and the way calculus approaches a solution. The meaning we focus on is related to the human practice of predicting values of a real magnitude that is changing: volume, temperature, cost, position, etc. Thus, we do not intend to ascribe our research results only to the tasks involving digital technology. Besides offering our proposals for the teaching and learning of calculus in our own institution (and working with teachers to use it with their students), we have been publishing textbooks, chapters and journal articles to disseminate our proposals (Salinas et al. 2011, 2012a, b, 2013; Alanís and Salinas 2010; Salinas and Alanís 2009).

Related to this meaning for calculus learning, the development of a sociocultural perspective for mathematical education seems appropriate. We believe that the classroom interaction among knowledge, teacher and students can be re-conceptualized through the lenses of dialogic learning (Fernández-Cárdenas 2015). Talk-in-interaction is no longer limited to an analysis of transmission of experiences from an expert to a learner. Today, it is about exchanges between a teacher and her or his students, or between groups of students who are thinking together about a situation, not least in the presence of supportive digital environments.

Several investigations about the learning of calculus have examined the development of linked meanings for derivatives (rate of change) and integrals (accumulation). A serious lack in student understanding of the relations between the derivative and the integral is a problem related to the meaning and significance of the fundamental theorem of calculus (FTC). The introduction of digital technology allows different approaches, which look to technology as a means to an end (e.g. Thompson et al. 2013; Hong and Thomas 2013; Yerushalmy and Swidan 2012). The meaning of rate of change or of accumulation is illustrated by technology as a 
process that simulates the sophisticated understanding of the limit concept. There, the process of approximation of numerical values is acquired by exploration and examination of a digital simulation performed on a graph, the one representing the rate of change function or the one representing the accumulation function. However, in contrast, the approach we offer to this same concern in our work does not deal with limit processes. Instead, we provide a global image of both functions (namely, rate of change and accumulation), already constructed in their own coordinate system, and their linked behaviour governed by the FTC as part of the software infrastructure.

In this article, we describe a sequence of educational tasks supported by the use of specialized digital technology and our students' interaction with them. Our goal is to interpret this experience from a sociocultural and dialogic perspective and to characterize their mathematics learning connected to interaction with a dynamic digital technology. First, we describe some elements of the sociocultural and dialogic field that allow us to situate our work. Second, we describe the design and implementation of a pedagogical sequence of tasks, focusing on the use of the software for fostering dialogue. Then we discuss some study results, including the analysis of talk in interaction for a pair of students, in order to provide evidence of mathematical negotiation of meaning arising from the ecology of the classroom and also to contribute to the design and implementation of educational tasks strengthened by this theory of dialogue.

\section{Towards a Dialogic Role for Technology in Mathematics Education}

The presence of true dialogue in classroom is not a common experience. According to Mercer and Howe (2012), school culture promotes adherence to particular 'ground rules' that may limit the potential value of talk to build knowledge. The commitment of the teacher in the interaction with students has been associated with the production of 'closed' questions seeking for the 'right' answers. If we think about the mathematics classroom, this situation additionally fosters a limited perception of mathematics knowledge. The answers are already given, derived from mathematical procedures in an algebraic fashion, and this limits the students to a stereotyped performance. We believe that repeating those procedures with pencil and paper the times necessary will make them fit well when the stereotyped exam arrives. Our point here is to challenge if that is the learning we should foster. Our intention to consider a sociocultural perspective in the research of mathematics classrooms looks for a way to change this situation, and regarding a dialogic perspective, we should make clear that we need an open mind to what is mathematical knowledge and which kind of thinking reveals the immersion of students doing mathematics.

One way to think about dialogue in practice is through the lenses of conversation analysis (Fernández-Cárdenas 2015). Conversation analysts are interested in the study of the way participants construct social order through conversation. In other words, they can be seen as sociologists who assume that everyday social structure is a skilled accomplishment by competent actors. With this focus, they use recorded conversation as their sole source of data, data that they transcribe and analyze in a very detailed way as instances of social construction in interaction. 
The analysis is based on the different turns taken by participants where context and discourse construct each other through means comprised mainly of turntaking, sequential positioning and adjacency pairs.

Turn-taking proceeds as follows (Sacks et al. 1974). First, speaker-change takes place. Second, overwhelmingly one participant talks at a time. Third, despite this quite significant tendency, occurrences of more than one speaker at a time are common, although brief. Fourth, transitions from one turn to the next with no (or only a slight) gap and overlap are frequent. Fifth, there are turn-allocation techniques: the person currently speaking can select the next person (for instance, by asking a question of a particular individual) or the next speaker might self-select. Additionally, there are usually no prearrangements for a naturally occurring conversation: neither the order nor the length of individual speakers' turns is specified beforehand. Similarly with the length of the conversation, the topics that will be discussed, the number of participants and the relative distribution of turns, none of these is pre-set.

Sequential positioning (Heritage 2001; Schegloff 1992) refers to the fact that turns talking are overwhelmingly produced both as a response to the preceding talk and as an anticipation of the kind of talk which is to follow. By formulating their present turn, speakers show an understanding of prior action and reveal their expectations about the next turn to come, at a multiplicity of levels. For instance, by accepting a turn, a participant can show an understanding that the previous turn was possibly complete, that it was addressed to him/her (or them) and/or that it was an action of a particular type (e.g. an invitation).

The notion of adjacency pairs arises from the observation that turns in a conversation minimally come in pairs (Levinson 1983). In this respect, the first turn generates specific expectations that restrict the possibilities of allocation for the second turn. For instance, adjacency pairs can occur in paired turns when performing question/ answer, complaint/apology, greeting/greeting, accusation/denial, etc. In this respect, pairs can be characterized as a preferred organization. Nevertheless, the occurrence of 'dispreferred seconds' has been also studied by researchers, who have documented this type of response in several different social situations. For example, it has been found that for a first turn expressing a request, the preferred second turn would be compliance, while the dispreferred one would be a refusal.

In this article, we will be using the previous three notions to analyze how a given event might be involved in constructing a dialogic interaction. This is because, as Fernández-Cárdenas (2015) has asserted, the sequence of turns in dialogue follows, in essence, the same spirit of the regularities identified in the tradition of conversation analysis.

\section{Conversations in Classrooms}

School curricula invoke disciplinary conversations (with their extensive and intricate histories), so that learning mathematics (among other subjects) can be considered in terms of being able to insert yourself into the conversation (a conversation that is part of a community with its own values). Digital technology is nowadays part of this conversation in mathematics, and in the spirit of dialogic education, we could take advantage of this fact to provide students with the opportunity to position themselves advantageously with regard to mathematical knowledge. 
Studies in education exploring a dialogic perspective have highlighted the importance of positioning language in the context of a relationship, as well as the ethics involved in a dialogic space, in which two or more voices can express themselves without trying to silence each other. Language only becomes 'our own' when we appropriate words, adapting them to our own semantic and expressive intention (Bakhtin 1982, 1986). Some researchers have proposed that dialogue could also involve interacting sequentially with other participants that might not be human. In this respect, Wegerif (2004) suggests introducing computers into educational conversations, given that computers have an ambivalent 'ontological' nature, acting simultaneously as subjects and objects. Unlike objects, subjects are considered to possess psychological attributes, such as thoughts, feelings and beliefs. Features like 'patience' and 'non-judgement', whose presence we could claim when interacting with software, could prove beneficial for learning. Educational software in a computer could be considered as a partner in learning conversations. This difference between computers and humans can be exploited in order to make the right educational software play "a unique role in supporting teaching and learning dialogues" (p. 180).

In this article, we identify this opportunity with the conception of an ecological environment in classrooms, one that promotes dialogue. From this perspective, students work collaboratively and dialogue is related to mathematical language that acquires meaning through interaction with dynamic digital technology. We care for students' appropriation of a mathematical way of speaking, as part of a culture where meaning is associated with mathematical concepts. We are certain that perceptual meaning is something that the history of the development of mathematics presents, although this has been overlooked in formal education. We believe that a dialogic education should allow us to recover such meaning when we do mathematics.

Moreno-Armella and Sriraman (2010) discuss the idea that mathematics is a human activity and that the mode of existence of mathematical concepts is semiotic. When we use symbolic representations, there is a mediating role, the symbols providing a means through which we can perform mathematical actions, such as multiplying, equationsolving or graphing. As Moreno-Armella and Sriraman assert: "computers have made feasible a new way of looking at symbols, looking through them, and transforming the resources of mathematical cognition" (p. 215).

Moreno-Armella et al. (2008) analyze the evolution of symbolic thinking over time. They state that a system of external symbols could act as a meta-cognitive mirror, in the sense that one's ideas about some field of knowledge can be socially shared: "then we can see our own thought reflected in that 'system' and discover something new about our own thinking” (p. 101). According to Hegedus and Moreno-Armella (2010), certain software allows interaction with mathematical representations in a dynamic way due to specific characteristics embedded in its infrastructure. These authors describe interaction as being a process that is bi-directional and is sustainable due to embodied features of the software.

Similar to a dialogic perspective, we find in computer software the potential to engage students in mathematical activity. The mathematical mastery of a software environment can sustain an intentional attitude from the student to understand the computer output following his/her input. This way we find possible to attain some level of appropriation of mathematical reasoning from the software mathematical 
infrastructure. Thus, it is important to analyze and redefine the kind of exploration space that could bring this to the fore.

Moreno-Armella and Hegedus (2009) use the term co-action to describe the way a user in a dynamic environment both guides actions occurring within it and is simultaneously guided by the environment. With this term, fluid activity between digital technologies and students could be discussed and modeled. It seems reasonable to ask how we could arrive at the level where students think through mathematical semiotic systems provided by digital technologies as similar to the way an experienced reader 'reads through the text'.

\section{SimCalc MathWorlds Fostering Dialogue}

SimCalc MathWorlds ${ }^{1}$ (hereinafter 'SimCalc') affords dynamical manipulation of Cartesian graphs that are linked to motion simulations taking place on a straight line. Since its conception, James Kaput (1942-2005) wished to address a semiotic perspective on mathematical notation. SimCalc allows pedagogical design through the construction and editing of documents, giving rise to scenarios to promote exploration, generate conjectures and support inference. As Burke et al. (2013) report, designers of SimCalc included researchers, professors and software developers. Their focus was on curriculum improvement by means of tasks and actions that would not be possible without technology. A main aspect of interface design is the representational infrastructure allowing the showing or hiding of different mathematical representations: graphical, algebraic and numerical.

SimCalc tasks vary according to the purpose of the researcher: we propose to focus only on graphical representation, including the simulation of a cartoon character. We are concerned with a sequence of tasks to promote the visual awareness of the behaviour of a function through its derivative. This means that, having seen the derivative's graph, the learner could come to visualize the behaviour of the function's graph. Linear motion is the first scenario where we worked with students: the function (accumulation) refers to the position, while the derivative (rate of change) refers to the velocity. Both magnitudes (position and velocity) are functions dependent on the magnitude time.

Figure 1 shows the original design SimCalc offers, as well as the scenario design we used with students and tasks related to linear motion. The order we propose responds also to the design: first velocity, then position. The possibilities for dynamic interaction with the SimCalc document are shown by the three dragging options included. There we could identify an intentional dragging in students associated to the three options given.

Dragging practices in Cabri environments have been analyzed by Arzarello et al. (2002), including the idea of dragging supporting the production of conjectures and identifying invariants. Differences with SimCalc infrastructure lead us to identify our idea of intentional dragging with the modality they call as guided dragging, namely "dragging the basic points of a drawing in order to give it a particular shape" (p. 67).

\footnotetext{
${ }^{1}$ SimCalc MathWorlds is a registered trademark of the Kaput Center, University of MassachusettsDartmouth.
} 


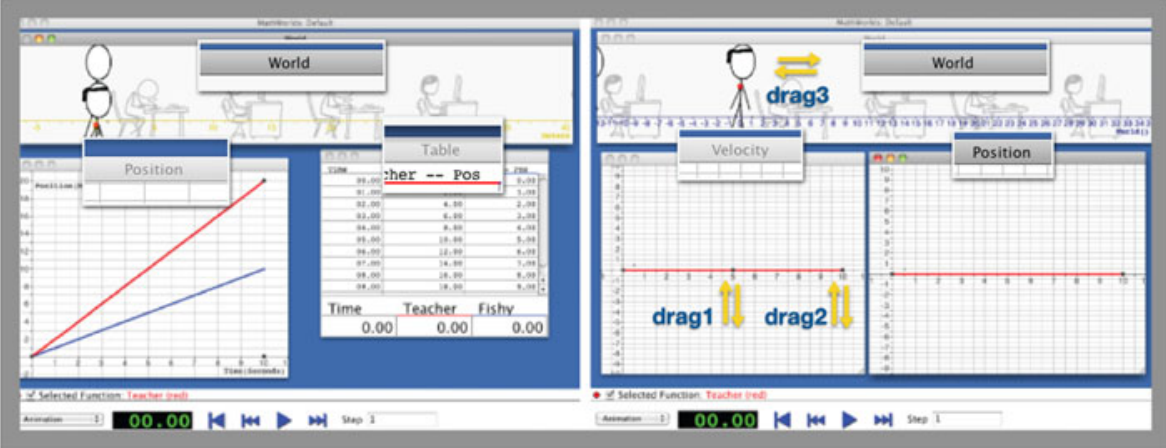

Fig. 1 Design for the SimCalc graphical interface with three options for dragging

Our learning purposes relied on working with SimCalc, because the FTC is incorporated into its programming. This means that any alteration performed to the velocity graph will provide instant feedback in the position graph, and vice versa. Students exploring this SimCalc document could drag the velocity graph, and its dynamic interaction is linked to the position graph, even when not in a student's mind.

In Fig. 2, we show the effect obtained for the two dragging options available for the velocity graph and the corresponding drag effect that is depicted in the position graph. Intentional drag 1 causes a shift up or down the same horizontal velocity line: because of the FTC acting internally, the corresponding position graph (a straight line) changes its slope. Intentional drag 2 causes a change in the slope of the velocity graph (a straight line with initial value zero), and the FTC provides a parabola for the corresponding position graph (a change in form). It is important to say that the formal version of the FTC is not the learning goal we are looking for: in contrast, we are looking for students to perceive and interpret some results that we get as a consequence of that theorem in the real context of motion.

It is also important to emphasize that we are taking advantage of the action of intentional drag of the velocity graph to obtain a reaction from the software that is depicted in the position graph. In this way, students could carry out some coactions by means of the software, trying to figure out visual changes in the position graph linked to their intentional action performed on the velocity graph, by selecting the three different drag options.

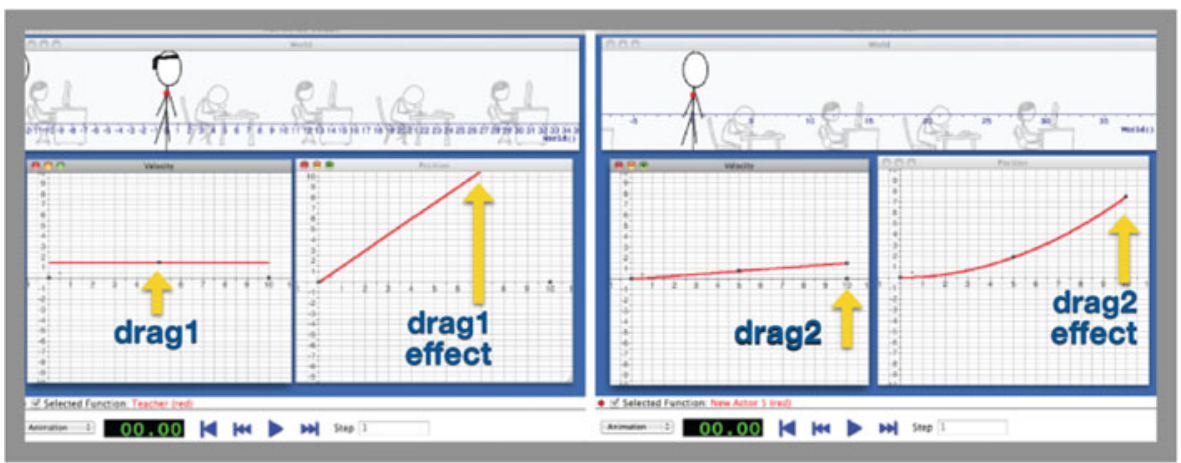

Fig. 2 The two drag options used on the velocity graph and its effect on the position graph 
In Fig. 3, we display several pictures assembled to show each type of dragging (1 and 2) on velocity graphs and its corresponding effect on position graphs. As you can see, getting to know the functioning of SimCalc document, it is possible for students to elaborate on intentional actions by using each of the drag options. They know that they could keep horizontal the velocity through drag 1, and this horizontal feature is an invariant. At the same time, the drag 1 effect also allows the depiction, as an invariant, of the straight-line behaviour of position graph.

The drag 2 scenario shown in Fig. 3 offers the straight-line behaviour of the velocity graph as an invariant, albeit a line changing its slope. Corresponding to this, the behaviour of the parabola of the position graph could allow several conjectures. There is also an extra feature (the drag 3 option), which could make conjectures to be analyzed through the additional change of the initial position on the cartoon. In addition, students have the opportunity to play the motion performance and see the cartoon moving to the right (or the left) and doing this progressively faster (or slower). Figure 4 shows the drag 3 option and its effect, leaving invariant the velocity but showing that the position could take positive or negative values, or even both.

Moreno-Armella and Sriraman (2005) discuss the necessity of conceiving new ways of thinking about meanings that students develop. Students can first develop situated observations inside the technological environment through exploration. The word situated reflects the role taken by the environment that includes the computational tool and the task being undertaken. The observations can refer to a certain property or result being expressed through the technological tool, where the environment facilitates its detection. This leads to the notion of a situated proof, the result of a systematic exploration intentionally carried out inside a technological environment to 'prove' a mathematical claim or relation.

For our pedagogical sequence, such situated proofs must take place through the coaction and talk-in-interaction that students develop when interacting with SimCalc. Our aim is that they arrive at an awareness of the relationships between velocity and position graphs. To establish them, we require students to use mathematical language interpreting different features of graphs in a Cartesian coordinate system. In some way, we are experiencing with driving the zone of proximal development of the artifact (ZPDA) as stated by Hegedus and Moreno-Armella (2010). Our point is that the constraints of the artifact's capacity can be an opportunity for the pedagogical sequence design. For example, it is not easy to visualize a 'positive' or 'increasing' feature of a

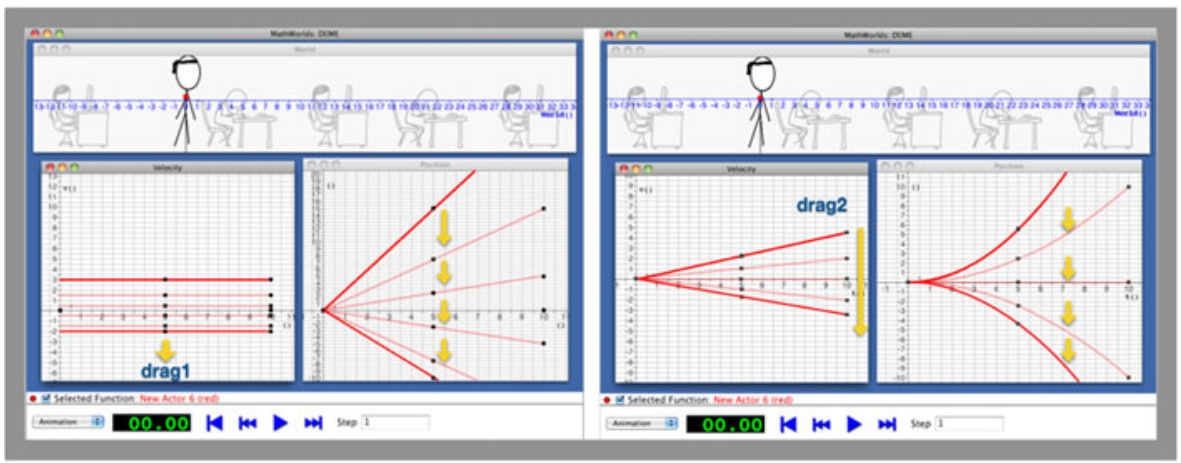

Fig. 3 A sequence of the two drag options on velocity graphs and the reaction on position graphs 


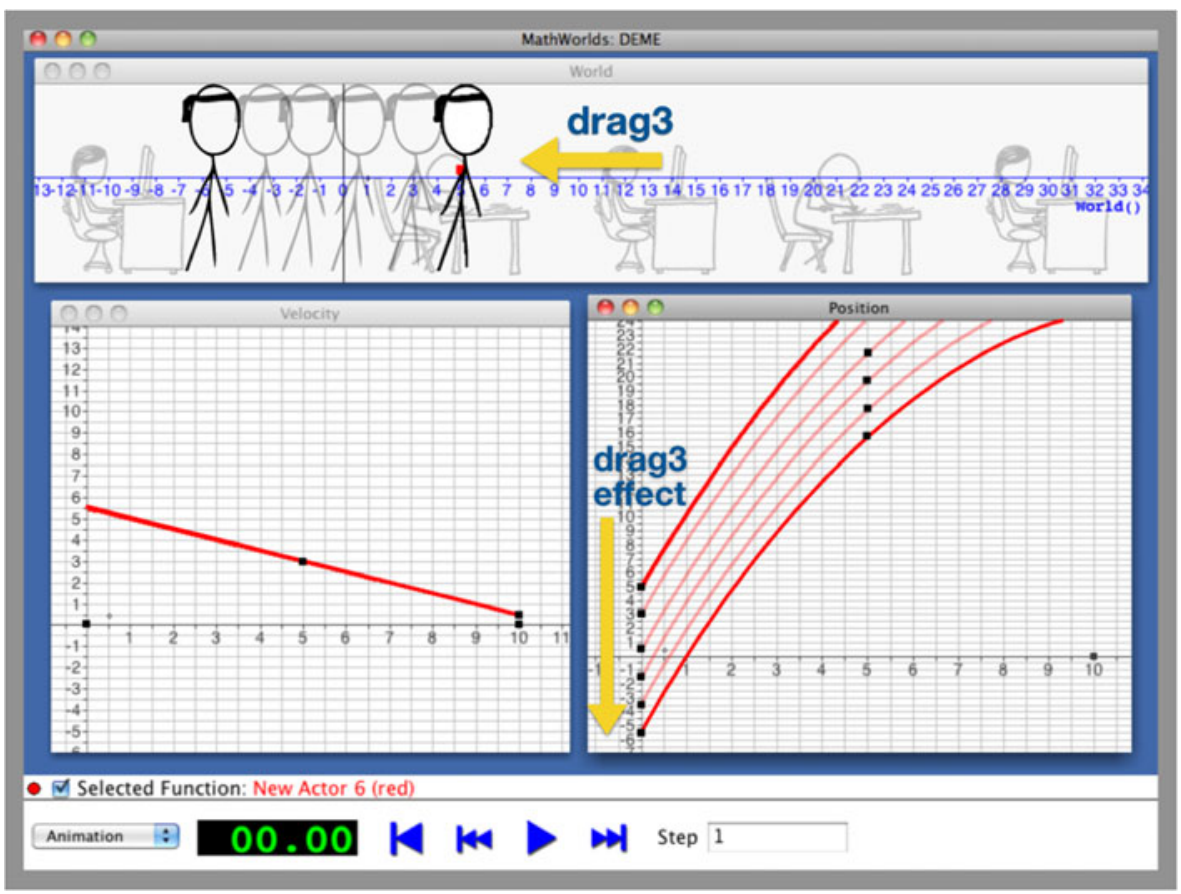

Fig. 4 The drag 3 option's effects on the position graph, showing several invariants

graph; yet it is part of mathematical reasoning that we must take into account. Those two words have different interpretations according to the context in which they appear but, as part of mathematical language, it is quite possible to misunderstand their meaning in terms of numerical values related to velocity or position.

We recognize two key elements for mathematical reasoning: generalization and symbolization. To make a generalization means to establish a statement that can be applied to multiple instances: thus, in order to generalize we must try to create a unifying expression that symbolizes all situations as one. In this sense, we could consider symbols as key elements supporting a generalization process.

We consider graphs as symbols representing particular information. Their features for example being a 'positive' or an 'increasing' graph - are ways to unify common behaviour. We use the word 'positive' in our natural language, but it refers to a generalization in our mathematical culture, and it is associated with some location. In the co-ordinate plane, 'positive' could refer to $x$ or to $y$ and, correspondingly, it should be located to the 'right' of an axis or 'above' an axis. We associate the word 'increasing' in our natural language with "growing or taking a bigger form". However, an 'increasing' graph should be interpreted simultaneously to the 'increasing' behaviour of numbers $x$ and $y$ relating to points on the graph. With these examples, we want to highlight the difficulties for students when dealing with graphical representations of functions.

Our design for the SimCalc document offers a global image involving the velocity and position graphs; this image could work as a symbolization of the relations between them. It is through the dragging of the velocity graph that students could observe 
changes in the new global image and perceive that some aspects remains invariant under this dragging. Through an intentional interaction with the software, it is possible to produce, eventually, a generalization expressing the relations between velocity and position graphs using words as 'positive' or 'increasing'.

In Fig. 5, we show (in a composite way) the intentional use of drag 1 over a velocity graph that already has a drag 2 effect carried out beforehand. These are the two possibilities for velocity behaviour and the position graphs show changes that combine increasing-decreasing behaviour with concave upward-downward graphs, and also with positive-negative location for position values.

Beyond the drag effect shown in last figure, we could execute the drag 3 option in the SimCalc document, which allows the visual perception of position graphs shifting vertically up or down. The combination of the three options for dragging makes us believe in the intentional interaction with the software by selecting each of them. Each time students execute one of the drag options, their action results in a SimCalcmediated reaction, and the visual perception of the global image including velocity and position effects, gives the opportunity for co-action to take place. Students could conjecture about the invariants found and continue with an active exploration through dragging, confirming or refuting their conjectures by participating in a dialogue between themselves and SimCalc as a way of producing a situated proof for their conjectures. Finally, they could establish them as generalizations, using mathematical language consisting of the opposite word pairs positive-negative, increasing-decreasing, and concave upward-downward. Within this context, we have been able to promote the use of hand gestures in students, paying attention to these visual and gestural events in order to bring students different ways for the appropriation of mathematical language (see Salinas et al. 2014; Salinas and Quintero 2013; Salinas 2013).

\section{Building an Environment for Dialogue Integrating Digital Technology}

Our contribution in this article is part of a broader research study where we used mixed data, mainly ethnographic in the qualitative strand, and with data obtained through exams (pre- and post-) in the quantitative one. Qualitative data included participant observation, video recordings of lessons and of students' laptop screens using SimCalc.

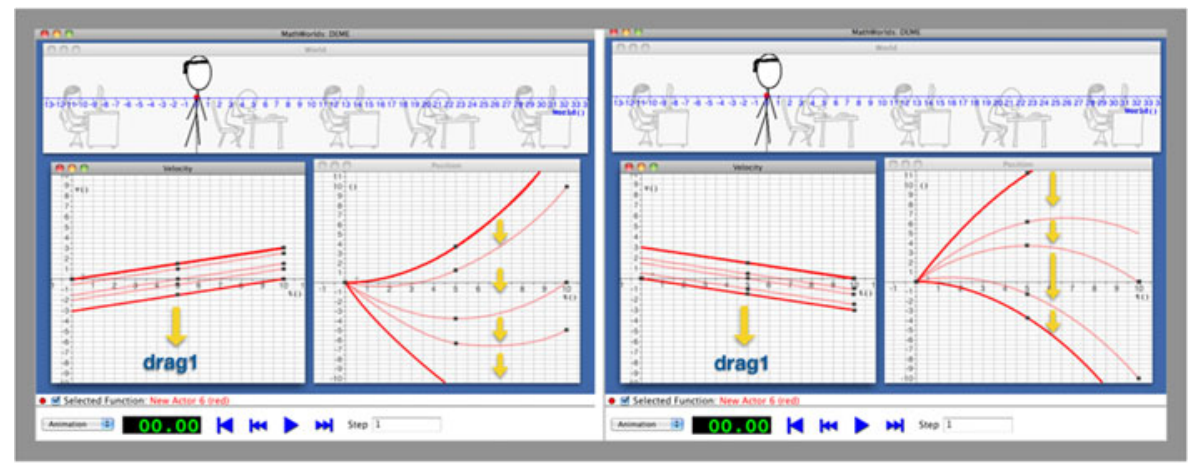

Fig. 5 The drag 1 effect performed over a drag 2 action executed before 
This way, students' talk and gestures are additional material for analysis, and semistructured interviews were performed to confirm analyses.

There were 60 students participating (all 19 years old approximately), who were part of three groups taught by one of the authors (Mathematics I for Engineering, spring semester, 2013), taught in a classroom that facilitated the use of technology and the collaboration of students. When the students worked in pairs, the organization of the classroom allowed the teacher to be supervising their work. Figure 6 shows the classroom organization for students working with some materials, and the active presence of teacher around the different groups of students. The technological platform (Blackboard Learn) facilitated making materials available for classroom use, and also communication between students and teacher.

The students were using one laptop per pair and were asked to videotape their interaction with SimCalc, producing a video of the laptop screen. We prepared technically for this asking them in the classroom to get a trial of Camtasia Studio 8. This additionally allowed them to record their faces and use of hands when doing the tasks. We collected and analyzed 28 video recordings, providing $3 \mathrm{~h}$ and $23 \mathrm{~min}$ of classroom data. Based on the 11 categories detected, and corresponding to 4 general domains, we decided to select six videos for transcription. The analysis of talk considered approximately 100 turns for each video. (See Section 5 for more details.)

Next, we describe briefly the whole sequence of tasks, but our intention in this article is to highlight the event of interaction with SimCalc. Thus, we will focus on the activity of peers performed during the third lesson, where we look for dialogue as an evidence of the event of co-action through SimCalc. For this paper we selected the work of two students to illustrate some results and develop our ideas about dialogue taking place in the mathematics classroom.

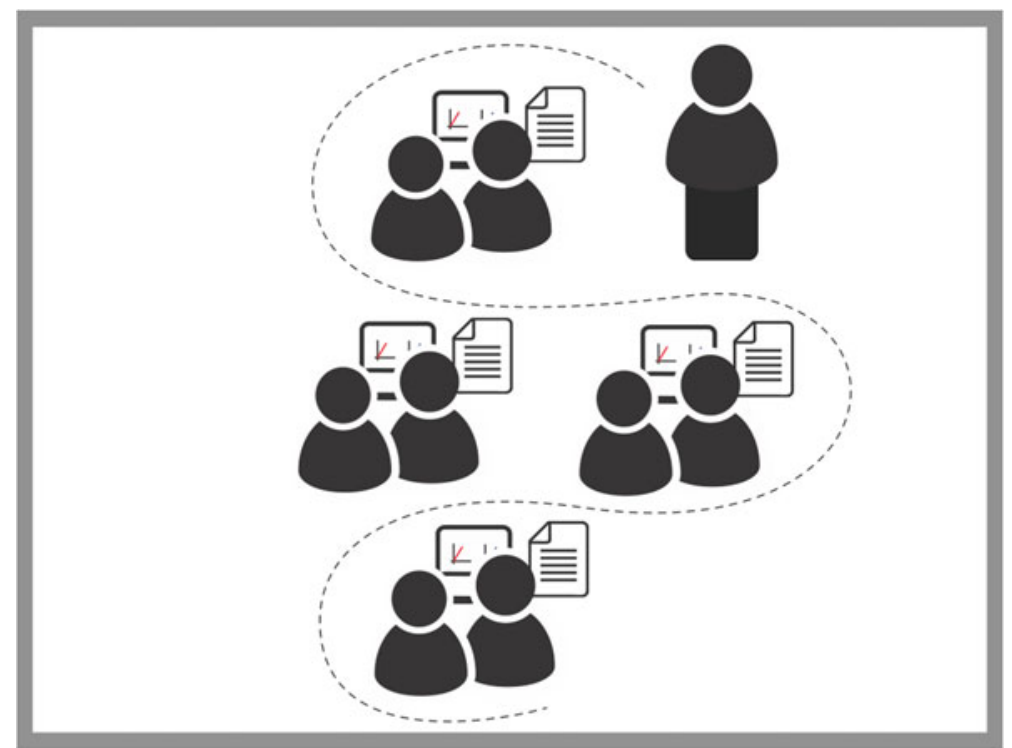

Fig. 6 Students in classroom worked in pairs with one laptop and one worksheet between them, with the teacher actively supervising their performance 


\section{Design of the Pedagogical Sequence}

The goal of the pedagogical sequence was to establish relationships between the velocity and position graphs, visualizing those features in the context of uniformly accelerated motion simulated in SimCalc. The design involved the organization of four lessons of 90 min each. The experience took 2 weeks of the academic schedule.

For the first lesson, the teacher interacted with a SimCalc document in front of the class, which was designed to identify and locate the maximum (or minimum) of a parabola. The intention was also to make students familiar with the software and have them realize that points on the graphs could be interpreted as values of time, position and velocity. The simulation of motion performed by the cartoon character allowed the class to discuss the representation of 'negative velocity' and identify this with linear motion to the left.

The second lesson involved the use of a SimCalc document by each student individually. Having been assisted with installing the SimCalc software onto their laptops including the SimCalc document used by the teacher in the first lesson. The goal was to identify the first two relations between velocity and position as requested on a worksheet, whose design invited use of the words in the boxes below whenever needed. There are sixteen possible combinations, even though just two of them are valid. Figure 7 shows the two correct responses.

Someone could conjecture a wrong relation like: velocity graph is increasing with position graph being positive. This is possible, because you can drag velocity in the SimCalc document in such a way that it remains increasing, while also maintaining its positive initial position. The student could think that this is a valid relation, but here is where an active exploration through the software could make a difference. The student interacting intentionally with SimCalc can identify that this relation is not an invariant because it depends on the dragging. This second task allowed students to become individually familiar with co-action with SimCalc and prepared them to explore with the dragging issues available in the document. At the same time, we assure that they

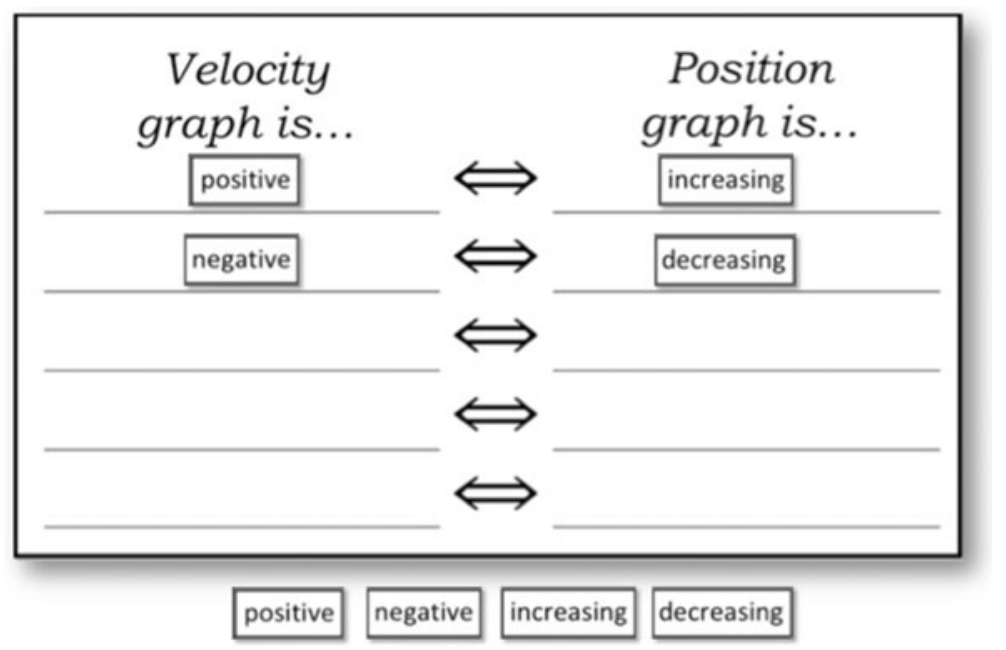

Fig. 7 Worksheet in the second lesson completed correctly 
already have the software installed and are able to access the SimCalc document in Blackboard Learn.

During the following lesson, they worked with peers. The third lesson is the one we focus on in this article. Here, students working with peers interacted with a SimCalc document designed to represent uniformly accelerated motion (and a worksheet to reflect their learning). Irrespective of the dragging they undertook (using all three drag options in order to probe their conjectures), there would always be a linear velocity graph. Supported by the two previous lessons, the intention of this task was to promote the visualization of the four relations between velocity and position graphs arising from this kind of linear motion. Figure 8 shows the worksheet filled out correctly. This image represents the knowledge achieved expressed in mathematical language which, at the same time, functions as a way to symbolize the mathematical generalization process we were hoping to foster. At the same time, it provided a channel for communication with the teacher about completing the task.

In the complete absence of meaning and context, each one of the blanks could be filled with six possibilities from the boxes; something that gives a lot of combinations that one should think to probe (36 in total). In addition, a mathematical graph combines the features given in the six boxes: for example, a velocity graph could be negative but also increasing. Each of these features could be associated with one feature of the position graph to make a conjecture. This kind of association represents a difficult situation cognitively, and it requires deep mathematical reasoning to look for the dragging that could verify whether the conjecture applies or not and, if not, what to change.

The fourth and final lesson in the pedagogical sequence was intended to review the results, providing an opportunity to listen to the students managing the mathematical interpretation of the graphs using language. It also allowed an invitation from the teacher to the students to employ drawing of graphs in the air with their hand to represent some particular mathematical behaviour. Those kinds of gestures were also observed during the third lesson when students were interacting with peers using SimCalc. This last class was supported through a class game we called 'the puzzle'.

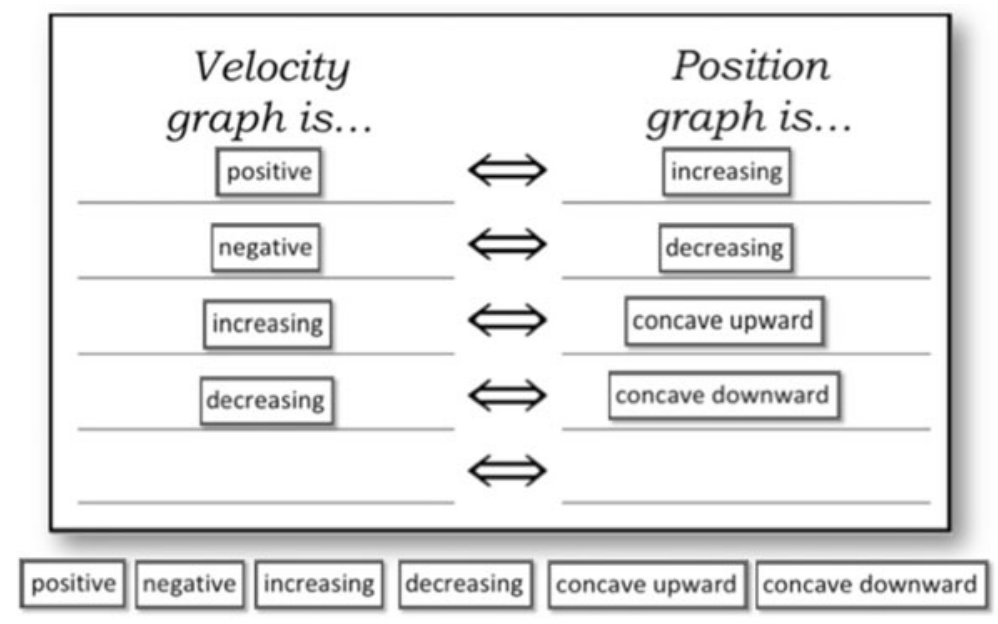

Fig. 8 Worksheet for the third lesson filled correctly 
The task, directed by the teacher, consisted of the visual association of a velocity graph with the corresponding position graph.

\section{The Third Lesson: Towards the Relations Between Velocity and Position Graphs}

As mentioned above, overall we identified eleven categories and four domains across the four lessons. In this article, we focus on the domain we termed 'SimCalc environment', involving four categories: co-action, situated proof, invariant search and exhaustive exploration. Providing complete information about the results is not the goal of this article, but even when all students delivered their worksheets with the four relations correct, each video analysis revealed different levels of talk in terms of these categories. Our intention here is to inquire after the presence of a dialogue event using mathematical language, promoted by the integration of SimCalc into the classroom environment. We relate the presence of dialogue to the presence of those categories: thus, we selected one of the videos that properly illustrates our point. The complete transcript included 104 turns.

At the beginning of the third lesson, the teacher introduced the task and recalled the mathematical meaning of the double arrow in the worksheet: a two-fold implication requires that any relation stated in their worksheet must work in any possible case for velocity and position graphs. Within the global image given by SimCalc, and by means of dragging, we expected students to identify certain invariants. This is possible because the software has as part of its infrastructure the FTC. In this way, we can be sure that, for instance, if the velocity is increasing, SimCalc always automatically associates it with a concave-upward position graph. This is an invariant that SimCalc affords and one that we were looking for the students to identify. Perhaps students did not focus on this feature, and wrongly associated increasing velocity with an increasing or decreasing graph position. This would result in them making a false conjecture. If they did not make an exhaustive exploration, the affordances of the environment due to the FTC would not be identified as invariants.

The students must identify the invariants, namely those features that do not change even with the changes produced by dragging actions in SimCalc. To identify those invariants, an exhaustive exploration is needed. The environment should allow this to be carried out, and that is what we were trying to capture. In class, they were simply told to fill in the blanks using only one box content. Students did not know if all the boxes were to be used, whether they could be repeated, how many rows should be filled or if they should add more rows.

Next, we present an extract of turns (in three separate parts) included in the transcript of the task carried out by two students, whom we call Daniel and Carlos. This was their first time working together. Judged by previous class experience, Carlos could be considered a student with a reflective attitude; he was more quiet and thoughtful. Daniel distinguished himself by being late for class, but he aimed to be proactive during the tasks. 
Daniel's laptop was used for the task, so Daniel took control of it and Carlos was in control of the worksheet. Before transcript 1 begins, Daniel and Carlos had already verified the two relations that were established in the previous lesson (positive velocity relates to increasing position graph and negative velocity relates to decreasing position graph).

Transcript 1. An initial exploration: "Well ... that's all, right?"

25. Eh, now when it is positive, is concave ... [Daniel uses the drag 2 option and the screen shows a Daniel: $\quad$ positive and increasing velocity graph and an increasing and concave-upward position graph]... is concave-upward, right? When it is positive Fig. 9.

27. Yes. Then when the velocity is positive, is concave-upward. [writes on the worksheet] Carlos:

28. Yes. Concave-upward.

Daniel:

29. And when it is negative, the position is concave-downward ... . [while writing on the worksheet] Carlos:

30. [Daniel modifies the velocity graph in order to obtain a negative and decreasing graph. SimCalc shows a decreasing and concave-downward position graph.] Fig. 10

31. Aha, when negative is concave downward.

Daniel:

40. Well ... that's all, right?

Daniel:

41. Well according to me, there is nothing else we can do. Because, for instance there, if we put it like Carlos: this, it depends .... [moves his hand up and down]

42. Because anyway if, how can I say, if we use this part [pointing at the screen] it is still the same. Daniel:

45. Aha. Well, that's all, right?

Carlos:

46. Yes ... Teacher, we're done. [raises his voice to address the teacher]

Daniel:

We observe Daniel interacting with SimCalc and setting the relations, while Carlos takes the role of writing down on the worksheet. They were looking for a third relationship and Daniel selected the word positive again. Daniel dragged velocity in such a way that the graph was still positive, but also increasing. The co-action through SimCalc displays a position graph that has three features: positive, increasing and concave-upward. Daniel (turn 25) asserted the position graph is concave-upward, and Carlos just played his role, repeating what Daniel said (turn 27) and wrote this incorrect relation in the worksheet. They continued in this fashion and established another incorrect relation for negative velocity (turns 29-31). It is important to notice that turn 40 ("Well ... that's all, right?") provided an opportunity to trigger a dialogue, because the question "right?" inherently reflects an intention to keep exploring, or at least to question possible new explorations. In turn 41, the laptop's screen with SimCalc shows an image that could have led the students to notice their mistake. 
In terms of conversation analysis, Daniel followed a preferred organization of adjacency pairs, by asking a question aimed at bringing the task to an end, and Carlos followed Daniel's remarks by remarking that "there's nothing else we can do". In this sense, the software plays a role in the dialogue, silent, but at the same time offering information to the students' visual perception. Because of this preferred organization of turns, they made no further effort at this point and their worksheet was filled in as shown in Fig. 9, with the two final relations wrong. They decide to ask the teacher about their work (turn 46).

Co-action with the software was not enough nor exhaustive as needed; their talk does not reveal active exploration. From the video, we noticed that Daniel used the drag 2 option and produced a positive velocity graph that was also increasing; they did not perceive this visually. Daniel looked at the concave-upward position graph and stayed with that information in mind. It is important to notice that if their exploration with SimCalc had considered velocity to be still positive but decreasing, which requires the use of an intentionally combined drag 1 and 2, they could be in a position to perceive the change in position graph to concave-downward. Without this intentional dragging being carried out, Carlos adhered to what Daniel says without an explanation.

For the second wrong relation, Daniel changed the positive and increasing velocity to be negative using a combination of drag 1 and 2, but the dragging performed resulted in the previously increasing velocity to become decreasing, as well as negative. They did not perceive this visually either and apparently just thought about the negative feature. Daniel observed the concave-downward behaviour for the position graph and related this to the co-action change from positive to negative velocity. Figure 10 shows the dragging sequence performed by Daniel: the numbers show a combination of the two drag options performed in such a way that the last velocity, in addition to be negative, is decreasing.

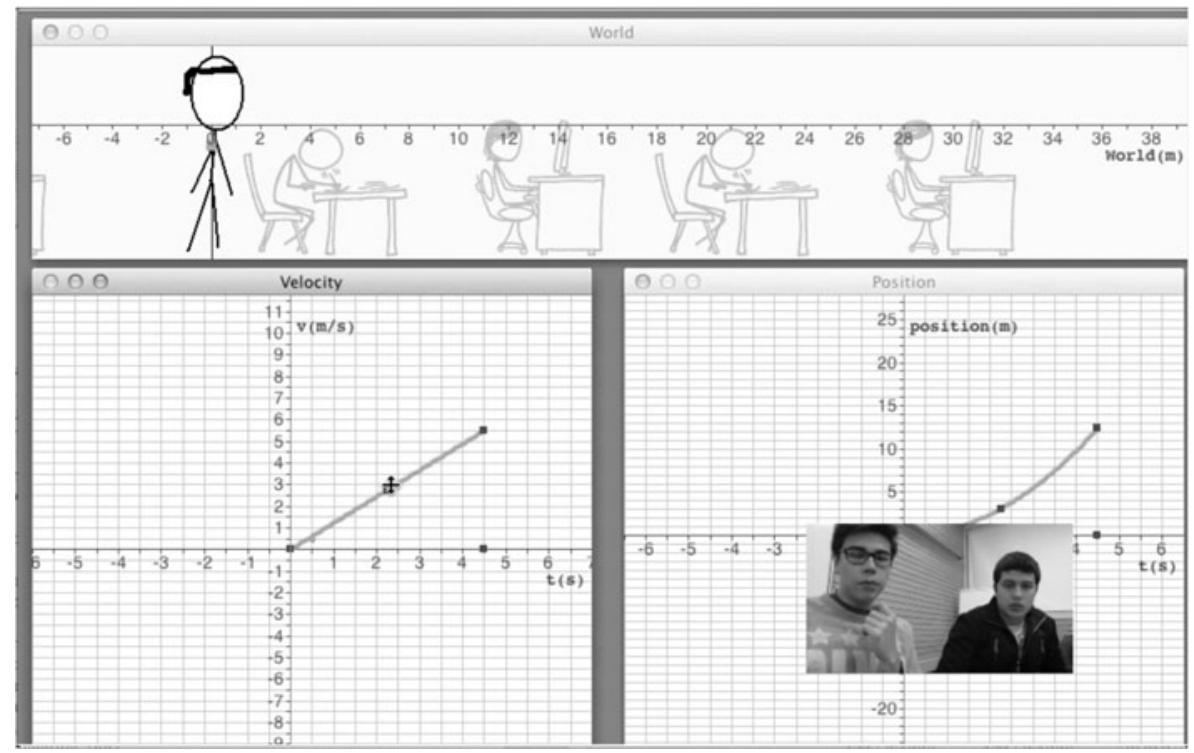

Fig. 9 Picture from the video in turn 25 


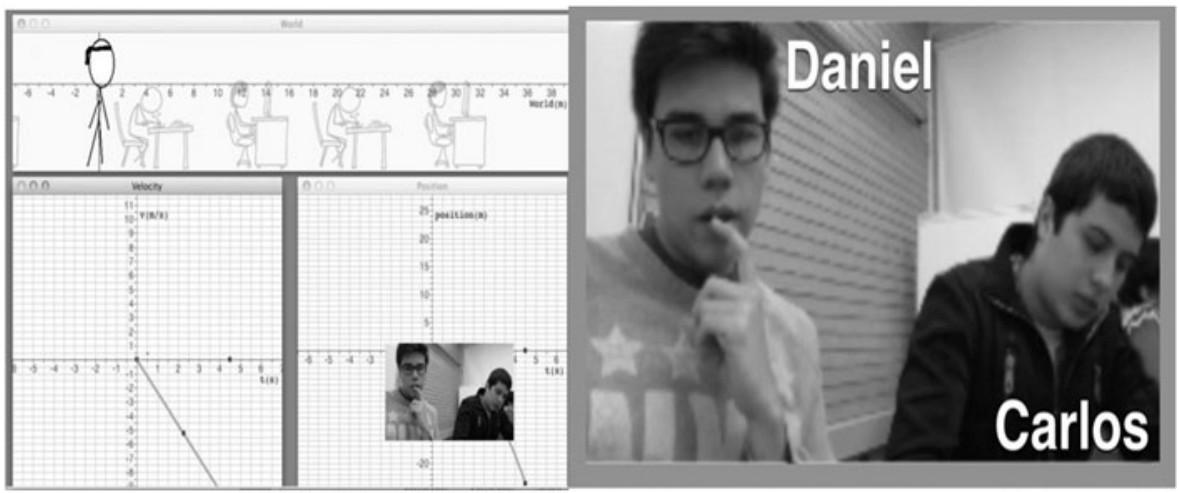

Fig. 10 Picture from the video in turn 30 and close up of students

Carlos looked closely to the screen, his face showed some doubt when Daniel used the drag 1 option again in step 6 in the last figure, as the velocity crossed the horizontal axis. But Daniel was already satisfied and attempted unsuccessfully to offer an argument, "Because anyway if, how can I say, if we use this part [pointing at the screen] it is still the same". Then their laptop's screen showed a decreasing velocity changing from positive to negative values. It was showing visually the wrong relation they wrote in the worksheet, but they failed to recognize it. As we can see, affordances are there, but this is not enough to arrive to the invariant identification. The students' talk was not as exhaustive or dialogic as the task required to be able to solve it.

Transcript 2. Correcting the mistake: "Yes, she's right. ... If the velocity is increasing, ..."

47. Teacher: [The teacher is coming towards them, looking at the worksheet.] Show me ... we're recording, right?

48. Carlos and Yes.

Daniel:

49. Teacher:

[The teacher has noticed the wrong relations in the worksheet.] OK. Show me one case where you have positive and concave-upward.

50.

[Daniel begins to modify the graphs displayed on screen. The image on the screen showed a decreasing velocity graph that changed from positive to negative values. Daniel used the drag 2 option changing the decreasing velocity graph to take only positive values. SimCalc then showed an increasing and concave-down position graph.) Fig. 14

51. Daniel: $\quad$ This is positive and this is concave upward. [Daniel directed his gaze to the teacher, while Carlos was looking at the screen carefully.]

52. Teacher: $\quad$ Ah OK, this you've got there...

53. Carlos: [Interrupts while looking thoroughly to the screen] It is concave-downward.

54. Teacher: Aha ...

55. Carlos: Instead, when the velocity is increasing, it is concave-upward.

56. Teacher: See? Here are problems, Carlos, but do not be satisfied: convince him about it, look at it through the software Fig. 15. 
57.

58. Carlos:

59. Daniel:

60. Carlos:
[Daniel changes the velocity graph to make it positive and increasing, as when they began work on the task, SimCalc showed a positive, increasing and concave-upward graph. The teacher left at that time.] Fig. 16

Yes, she's right [he orientates towards Daniel] ... If the velocity is increasing, this is positive ...

And it is growing ...

When growing, the position is concave-upward; because the last one was positive, but it was decreasing and it was concave-downward.

The teacher noticed that the last two relations in the worksheet were wrong. Without making this evident to the students, she asked them to show the third case in SimCalc where they had a positive velocity graph corresponding to a concave-upward position graph (turn 49). This turn provided a good opportunity for triggering a dialogic interaction. Daniel started a co-action by dragging the velocity graph up, but this time he was starting with a decreasing velocity graph and he used a combination of drag options (as shown in Fig. 11). His action did not change this decreasing behaviour (turn 50) and through co-action the software showed a positive and decreasing velocity graph. The position graph was not concave-upward this time, as they had thought before, and this made them realize their mistake Fig. 17.

Analyzing the video, it is noticeable that Carlos was watching carefully and interrupted Daniel to state his insight: "It is concave down" (turn 53). This is where SimCalc played its role in the dialogue, because the software expressed information in its own way, allowing the visualization that made Carlos aware of the situation. He immediately offered a correction in turn 55, saying, "Instead, when the velocity is increasing, it is concave-upward". He hastened to correct their worksheet, changing the word 'positive' for 'increasing' in the velocity graph column.

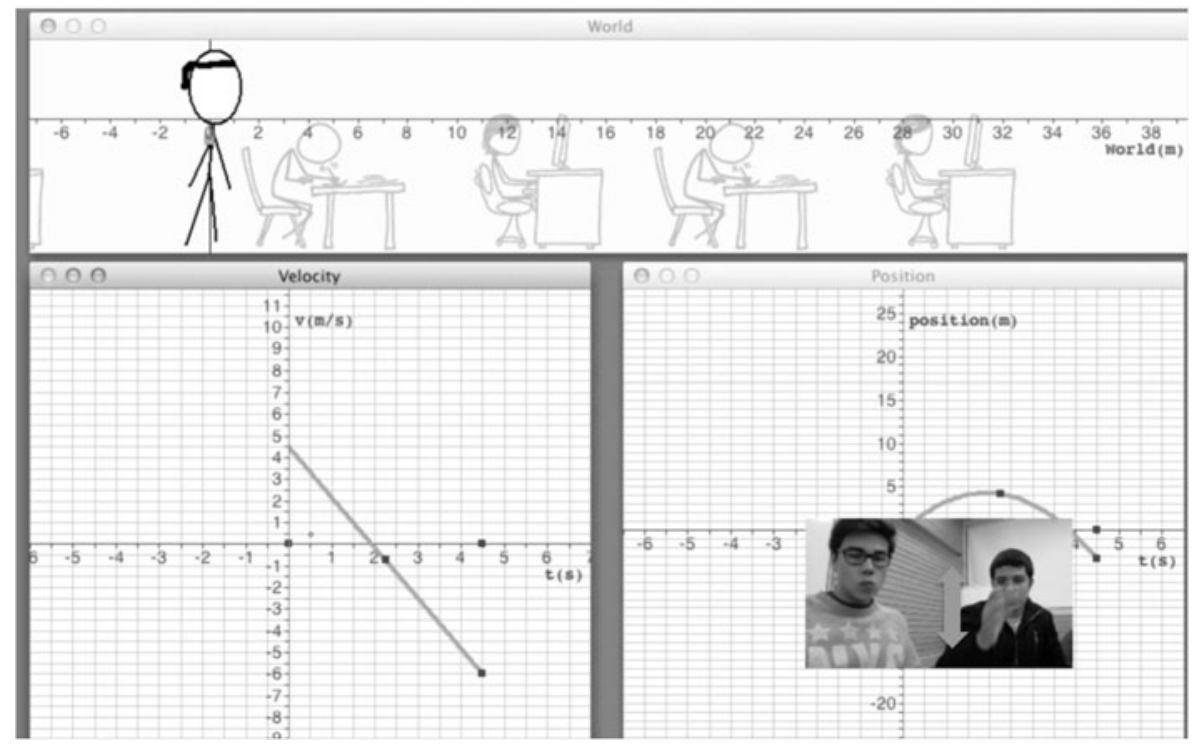

Fig. 11 Picture from the video in turn 41 


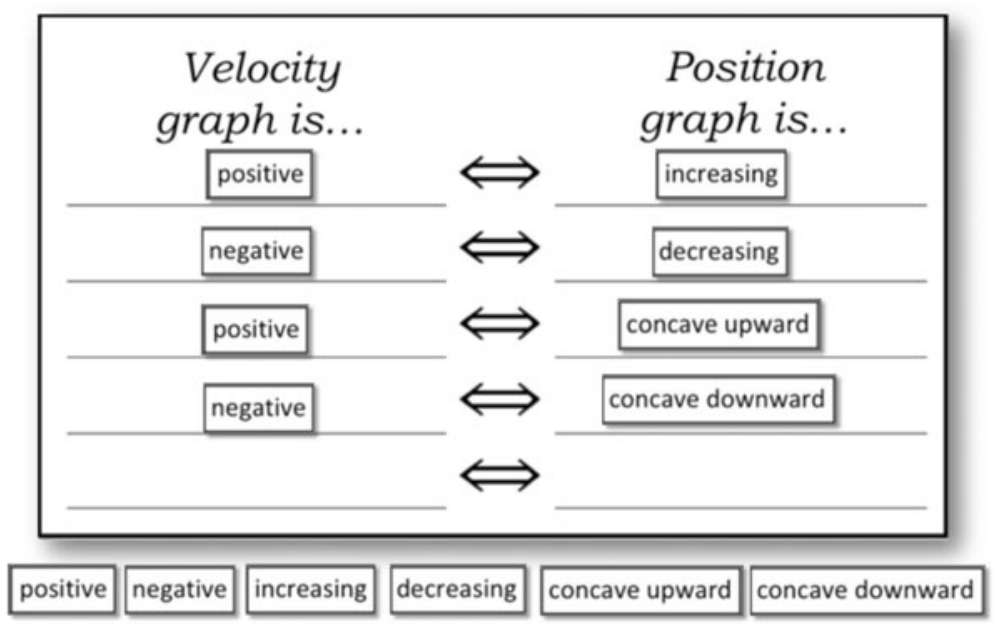

Fig. 12 Worksheet by Carlos and Daniel with the two final relations wrong

In conversation analysis terms, we would suggest that SimCalc triggered a rupture in the conversation which was repaired (Schegloff et al. 1977; Schegloff 1992) by Carlos in turn 53, because he had noticed a problem with Daniel's previous assertion (turn 51), as had been carefully highlighted by the teacher in turn 52. In other words, in turn 53, Carlos realized that when the velocity is decreasing, the position graph "is concave downward". Moreover, the conversational repair is doubled, as Daniel also strikingly stated the complementary relation of "when the velocity is increasing, it [the position] is concave-upward" (turn 55). The teacher perceived that Carlos has already the right relations in mind through that strategic interaction with the software, and leaves them

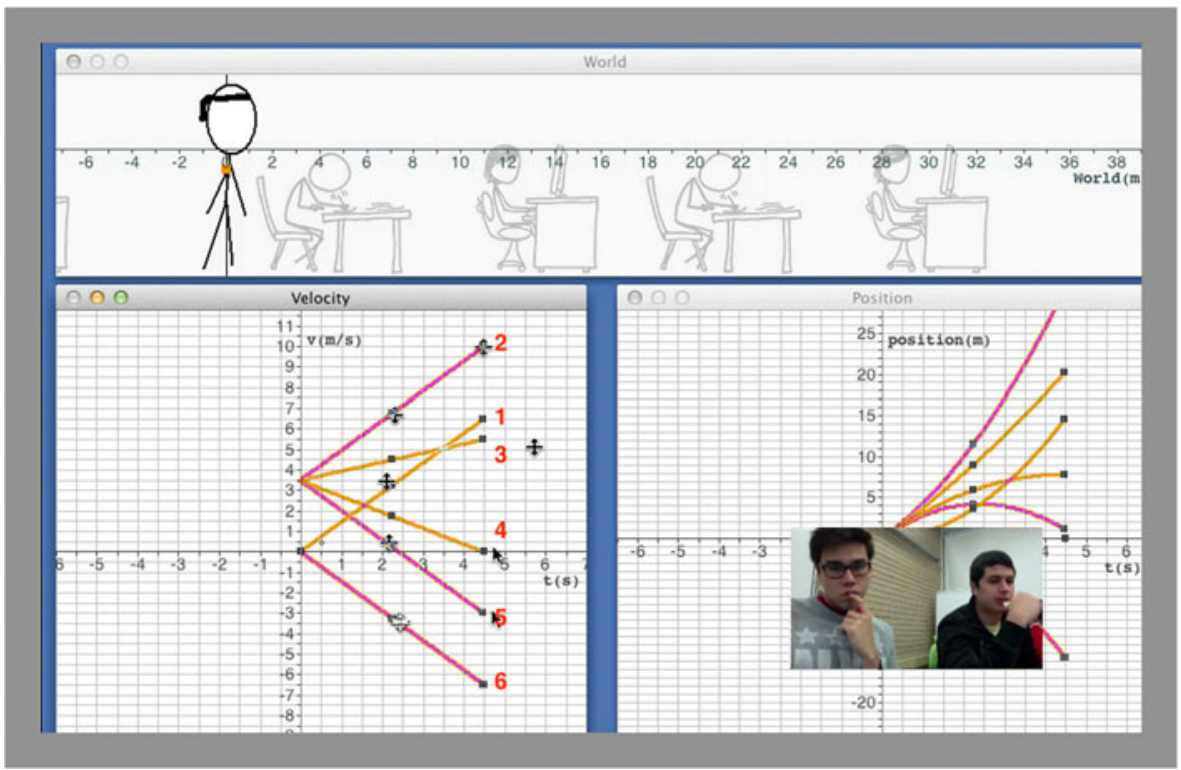

Fig. 13 Daniel uses the drag 1 option for steps 2 and 6, and the drag 2 option for the rest of the steps 


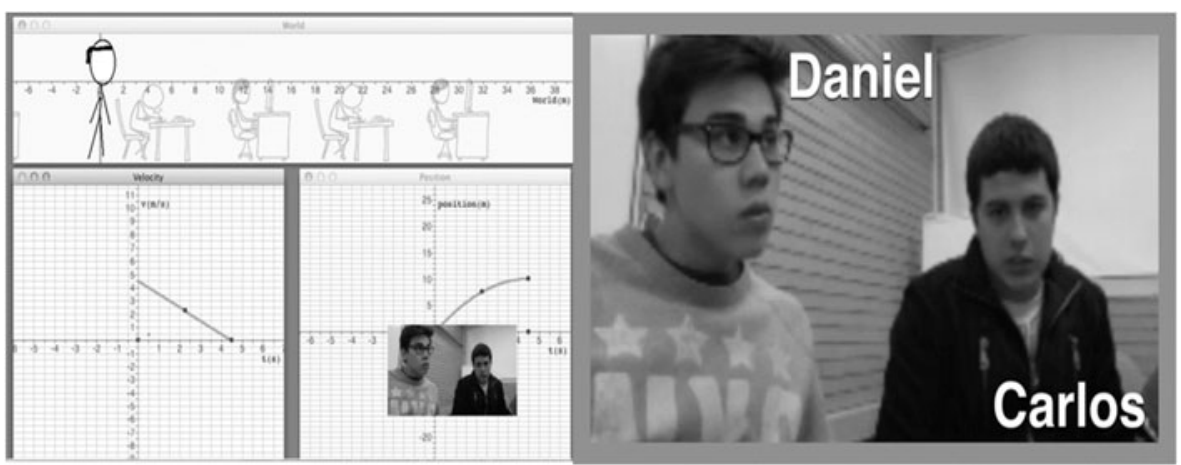

Fig. 14 Picture from the video in turn 51 and close up of students

asking Carlos to persuade Daniel using SimCalc. This intervention directs students to a dialogic interaction and an exhaustive exploration with the software ("convince him about it, look at it with the software", turn 56).

At that time, Daniel was already dragging the velocity graph with an active visual perception, because he was now able to return to the beginning of his actions looking intentionally to obtain what he was looking for: a positive, increasing velocity graph (turn 57). Carlos confirmed through that co-action with SimCalc that the velocity was positive (turn 58) and Daniel interrupted to acknowledge with some surprise, "and it is growing" (turn 59). These turns show them arriving at a suitable establishment of the relations between velocity and position graphs. The students' talk feels accompanied by the SimCalc co-action, showing a combined use of drag 1 and 2 options. SimCalc, Carlos and Daniel are in dialogue with words, with dragging and with seeing the co-action take place. The intentionality in the dragging sustains an exhaustive exploration and the

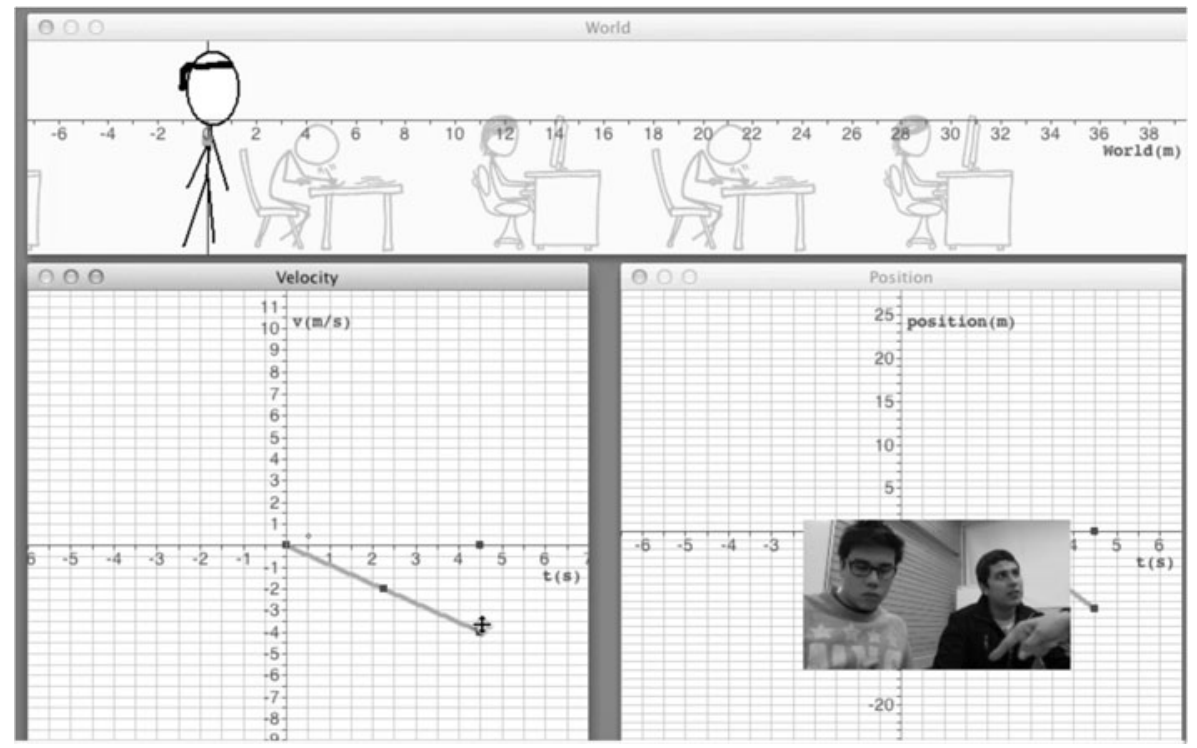

Fig. 15 Picture from the video in turn 56 


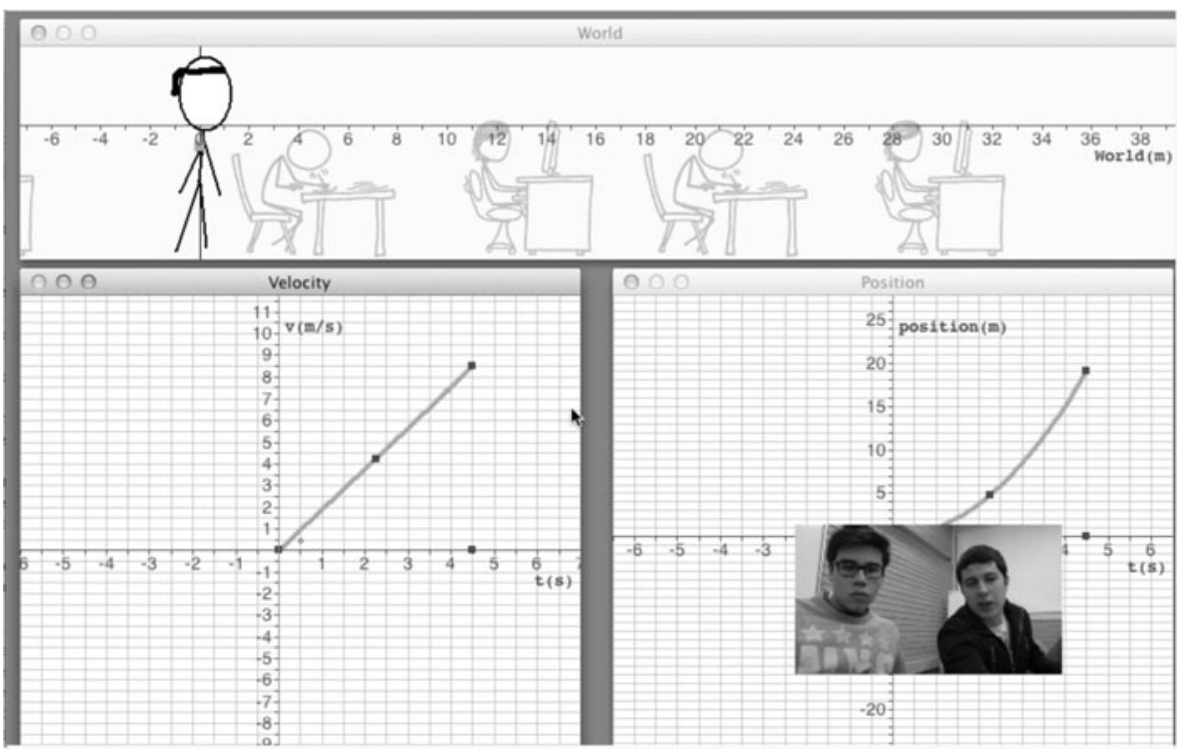

Fig. 16 Picture from the video in turn 58

affordances of SimCalc finally allow them to identify the invariant: meanwhile, the velocity graph is increasing, while the position graph is concave-upward. Carlos was finally capable of explaining the confusion they had had before (turn 60).

The exploration using SimCalc was not enough at the beginning. The teacher's intervention evidenced a plurality of perspectives that persuaded them to continue

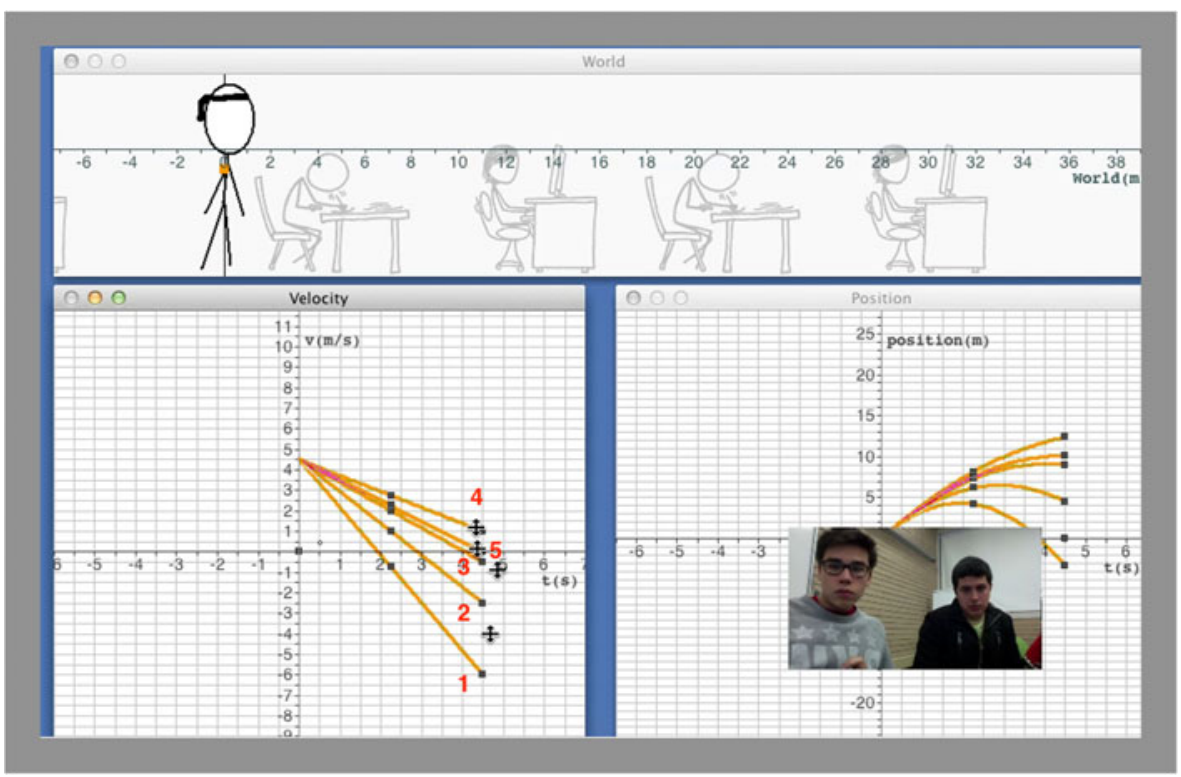

Fig. 17 Daniel uses the drag 2 option for all the steps, preserving the decreasing behaviour 


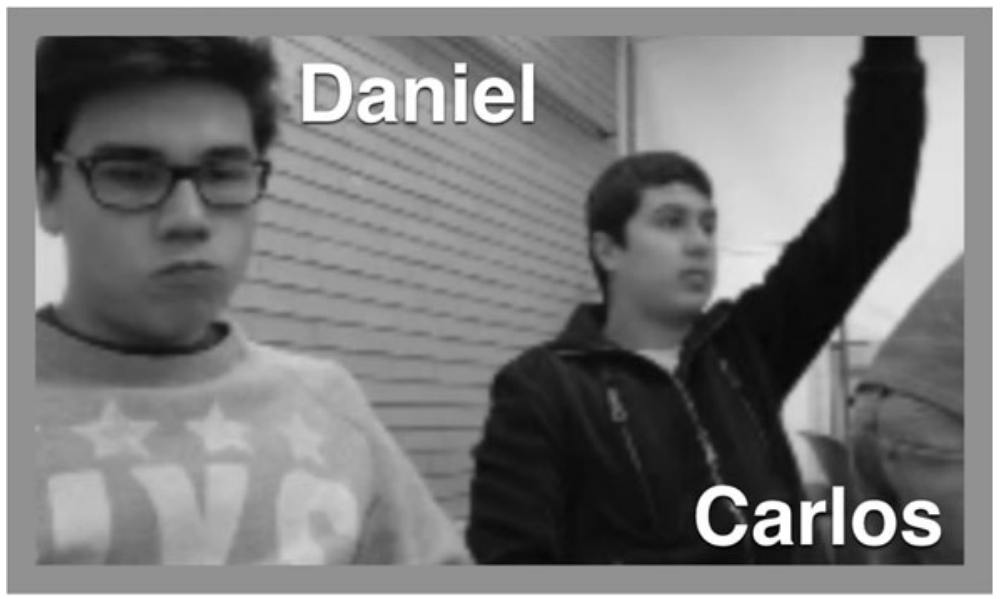

Fig. 18 Close up of the students during turn 81

and, at the same time, helped them to advance into the task. The intentional dragging prompted by the teacher made them notice that it was possible to have a positive velocity graph, but with different features, because it could be increasing or decreasing. These last two features affect the behaviour of the position graph. In turn 60, Carlos talked about the right relations between the velocity and position graphs, and he was ready to guide Daniel's exploration using the software in order to clear up the original confusion for themselves.

Transcript 3. An exhaustive exploration: "In both of the two axes will be concavedownward. And even though it is crossing them."

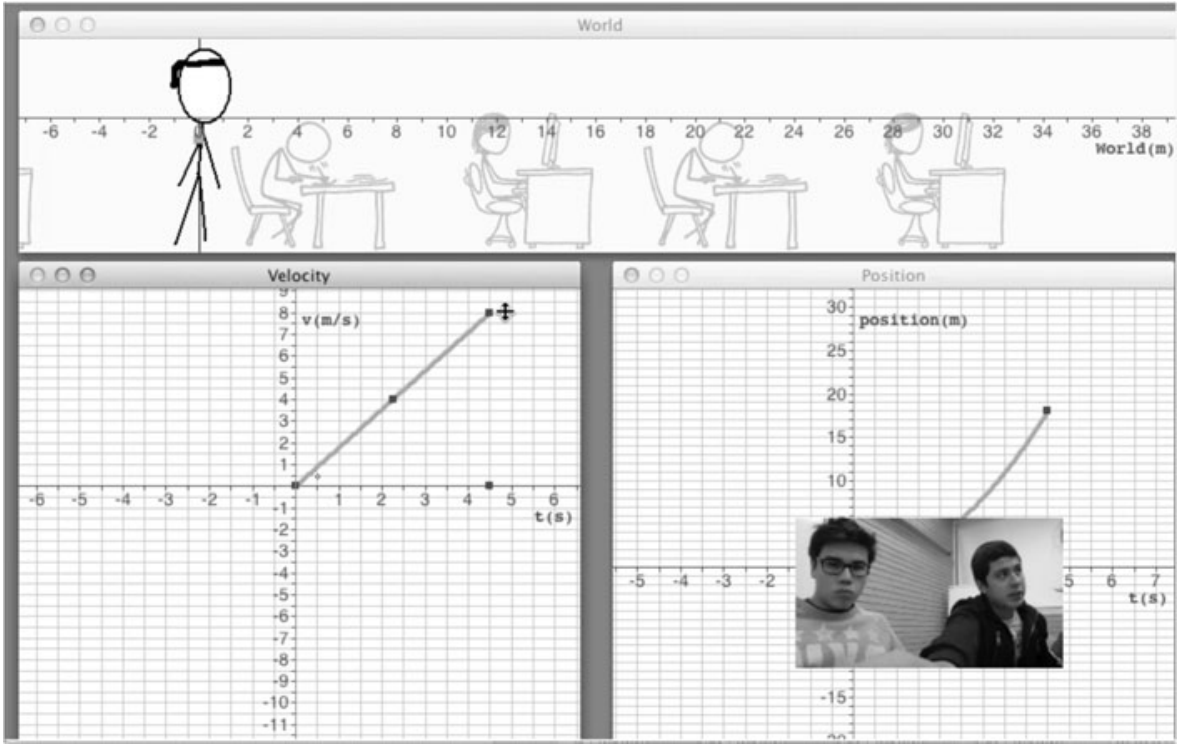

Fig. 19 Picture from the video in turn 90 


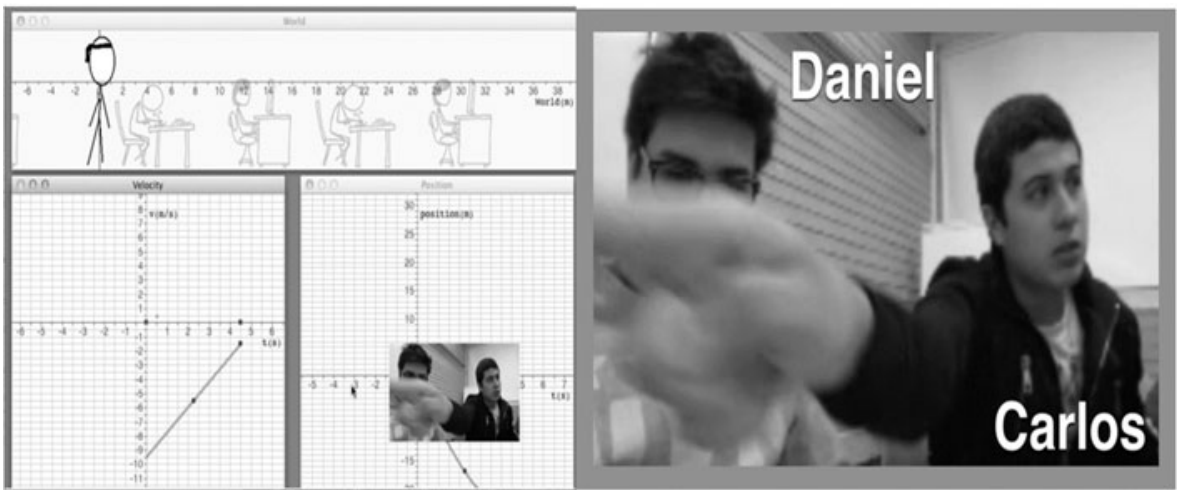

Fig. 20 Picture from the video in turn 91 and close up of the students

78. Carlos: So, I think now, these are the ones that can be.

79. Daniel: Yes, aha.

80. Carlos: Yes, that's it. We must tell the teacher ... to see what she thinks.

81. [Daniel continues reproducing in SimCalc the cases of both, positive and negative decreasing velocity. Carlos raises his hand and the teacher approaches.] Fig. 18

88. Carlos: Teacher, these are the cases we found. We think they are the only ones that can be. [the teacher looks at the worksheet]

89. Ah OK, and now it's OK ... Can you show me one that is increasing and concave-upward?

Teacher: Increasing velocity.

90. Carlos:

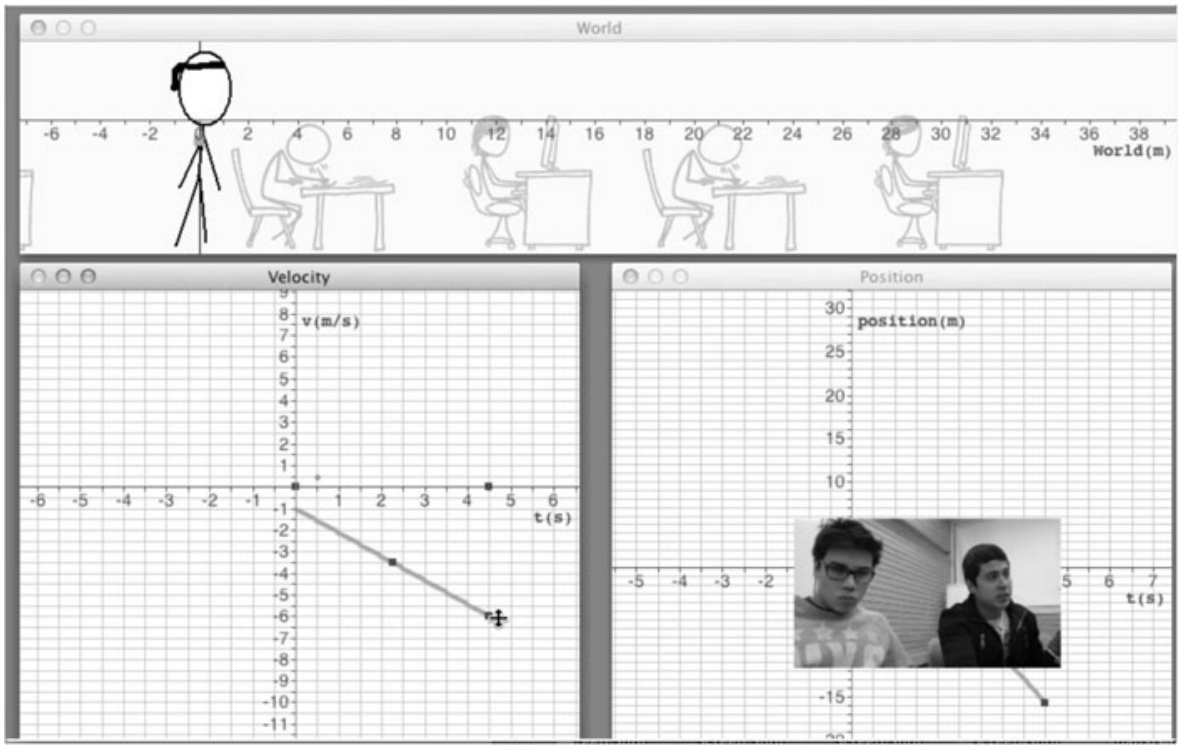

Fig. 21 Picture from the video in turn 94. Carlos: In both of the two axes will be concave-downward. And even though it is crossing them. [Daniel produces in SimCalc a velocity graph that takes both positive and negative values, while keeping the decreasing behaviour.] 


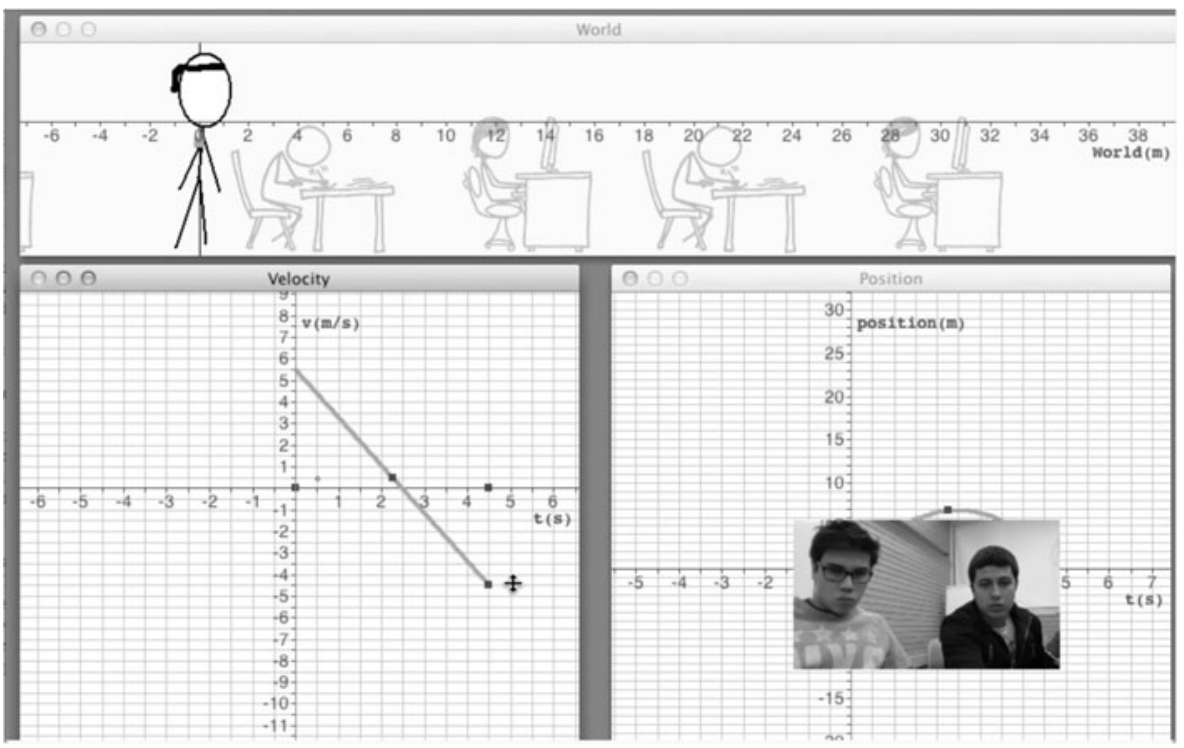

Fig. 22 Picture from the video in turn 94

Yes. [Daniel modifies the graphs as requested by the teacher, then produces a positive and increasing velocity graph, while SimCalc shows an increasing, concave-upward position graph.] Fig. 19

91. Carlos: There is increasing and it is concave-upward. [points to the velocity and then the position graph on the screen, then looks at the teacher] If we place it on the negative axis, [Daniel interacts with SimCalc to make the increasing velocity graph take negative values; SimCalc shows a position graph that is decreasing and concave-upward] it's also concave-upward Fig. 20.

92. It is also concave-upward. There, that's right ...

Teacher:

93. Carlos: That is, it is concave-upward. And if we make it decreasing ... [Daniel in SimCalc uses drag 1 option to make the velocity graph positive and decreasing.] Figs. 21 and 22

95. Even crossing!

Teacher:

96. Carlos: Even crossing the origin, still will be concave-downward.

Carlos and Daniel had their worksheet filled with the right relations between velocity and position graphs. They were ready to ask for the teacher's review. Carlos called her (turn 81) and the teacher verified the worksheet was right. In turn 89, the teacher promoted a dialogic interaction again and asks them to explain the first relation they previously had wrong. Carlos and Daniel gave the teacher a synchronized rationale for their active exploration through SimCalc (turn 90). Figure 12 shows the sequence of intentional dragging performed by Daniel that accompanied the explanation given to the teacher by Carlos. Note his intentional dragging from a positive and increasing graph to a positive but decreasing one Fig. 23.

The intentional dragging performed this time shows control of the 'dialogue' with SimCalc. The video shows that Carlos was talking and, simultaneously, Daniel was following his speech, while dragging to recreate and mirror Carlos's explanation in the 


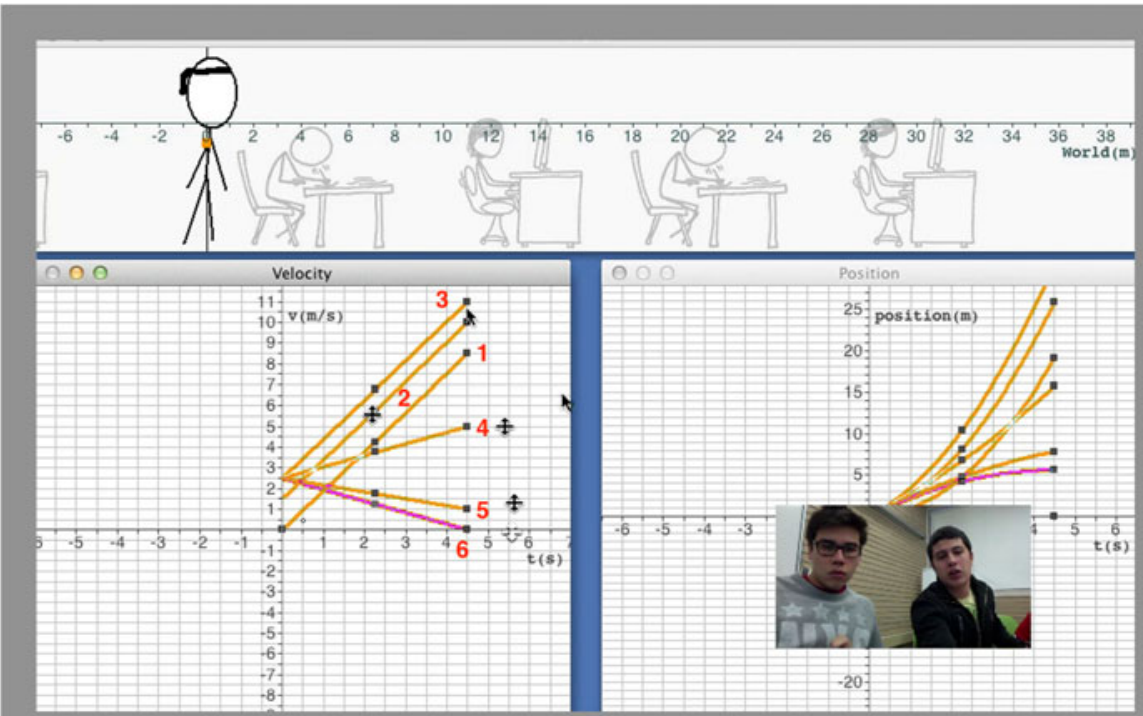

Fig. 23 Daniel follows Carlos' talk using drag type 1 to step 3 and then using drag type 2 to step 6

right place (turns 91 and 93). The co-action, possible by the use of SimCalc affordances, was recreated by Daniel while Carlos talked. In front of the teacher they were able to argue for their appropriation of the correct relations between velocity and position graphs by interaction with SimCalc.

By turn 94, they had arrived back at the same global image they had originally had in turn 41, when Daniel called the teacher for the first time. But now they could explain that situation correctly to the teacher. The decreasing behaviour of velocity graph was the reason for the concave-downward behaviour of the position graph. They noticed positive and also negative values for velocity; but even when the velocity graph crossed the axis, its important feature was its decreasing behaviour, which is why the concavedown behaviour of the position graph remains as an invariant. The video showed some excitement when the students were keeping the decreasing feature of the velocity graph in mind (even if it is positive or negative) related to the concave-downward feature of the position graph (whether increasing or decreasing). They finally completed their worksheet with the correct relations between both graphs, and showed its appropriation through synchronized talk and dragging through SimCalc. The teacher interrupted in turn 95 showing surprise "Even crossing!" and Daniel and Carlos proudly confirmed their learning in turn 96.

In terms of conversation analysis, the sequentiality of turns, guided by the questions of the teacher, afforded a systematic and more exhaustive exploration of the conditions presented in this task mediated by SimCalc. According to Goodwin (2007), through the unfolding sequences of events shared by the participants, they are capable of become attuned to what is at stake moment-by-moment, eventually reaching a shared understanding of the situation. In the example provided here, the students were capable of tuning in to the perspective of the teacher, who was carefully scaffolding the presentation of alternatives not previously considered by her students. Dialogue includes, 
then, not only collaboration between students, but also the presentation of their work to their teacher, who can then assess and provide feedback on their performance appropriately. Dialogue is also about giving learners the opportunities required to share their work with their teacher, in order to engage in meaningful conversations, which can eventually transform their own understanding.

\section{Discussion and Conclusion}

In this article, we reviewed theoretical elements related to dialogic education, and also to mathematics education. We also addressed a perspective of mathematics knowledge associated with real-world meaning as a suitable way for learning mathematics. By means of the design of a didactic sequence, we focused on students' appropriation of a repertoire of mathematical language, consisting of common words that are relevant to the interpretation of graphs. This repertoire allows students to talk mathematically about the behaviour of velocity and position graphs, and to describe features of the motion taking place.

In order to advocate for the presence of dialogue in calculus classrooms, we want to propose the perspective of considering multiple conversations taking place in the environment. First, we consider an inner dialogue between teacher and mathematical knowledge. Calculus, as part of a scientific knowledge, is a conversation that has remained through time. But analyzing its evolution we realize that, finally, this is a conversation among mathematicians who had to take care of a problem of theoretical foundations. Thus, a logical and rigorous structure organizes mathematical knowledge unrelated to a real-world meaning, and it is this side of the conversation which has remained in formal education. It is our claim that teachers should know about these facts in the evolution of mathematics and share a mathematical culture to support the learning of mathematics giving place to a dialogue with meaning.

Secondly, pedagogical design should also reside in the dialogue between teacher and digital technology. Today, dynamic software related to mathematics could be incorporated as a partner in our own thinking processes. Digital technology allows us to develop a mathematical reasoning in an enriched, visual way. It promotes engaging in an active exploration through the mathematical representations: numeric, algebraic and graphical. Above all, graphs are now available as part of our digital culture, and it is a great opportunity to introduce dialogue in mathematics classroom through the interpretation of the behaviour that stands visually within our grasp.

The pedagogical sequence we presented here aimed to giving students an opportunity to engage in dialogue between them, and also between each of them and the computer, two more dialogic events taking place. SimCalc is a suitable tool to foster a co-action process and, through it, students are taking part in the dialogue with mathematical representations. Intentional dragging is an input for the students' production of mathematical conjectures. That is a quite important element of 'doing mathematics'. Once they have already something in mind to confirm, it is not just the testing of different isolated options with the software. Actually, an active exploration gives the opportunity to develop a mathematical reasoning. As was illustrated with Carlos and Daniel, they come to realize that features of two graphs are causally interrelated. The students' moving eyes are engaged in active perception, and the increasing feature of 
the graph becomes finally identified as the invariant that relates with the concave upward feature of position graph.

During the performance of the sequence of tasks, we noticed that the classroom functions as an environment where the different elements participate with mutual dependence. A key piece for this dialogic system is the mathematical language related to features that matter in the interpretation of graphs. Giving importance to a repertoire of mathematical terms has functioned as a way to maintain a dialogue with calculus. Figure 13 shows a representation of the classroom environment encouraging dialogue, where the teacher, mathematical language (via the worksheet), digital technology (tasks using SimCalc) and students all share an environment for the promotion of dialogue Fig. 24.

The pedagogical sequence aims to foster the appropriation of mathematical knowledge. Our research makes us realize the presence of several elements for this to happen, and also different paths for appropriation to take place. Carlos and Daniel are one of the 28 pairs, the one we chose to show the potential of an exploratory talk between students and software. In their case, the dialogue between them and SimCalc was not enough at the beginning, but their asking the teacher to review their work became a crucial element in the environment to promote an exhaustive visual exploration.

We could enlist several elements in classroom: teacher, students working in pairs, laptops with a SimCalc document, and worksheets involving mathematical language. But fruitful relations among them arise through the dialogic event in the environment. The ecological metaphor helps us emphasizing the mutual dependence among dialogues, beginning with the design and ending with the classroom implementation. In this sense, we want to end by highlighting the idea of a dialogic ecosystem in a classroom environment. With the data and transcripts presented in this work, we want to propose an ecological view of dialogue in the classroom for the learning of mathematical knowledge through dynamic digital technology.

Our idea of dialogic ecosystems for mathematics education makes us emphasize the intentional interaction of students working with dynamic digital technology. Technology affords the mathematical representations and an active exploration reflects in the

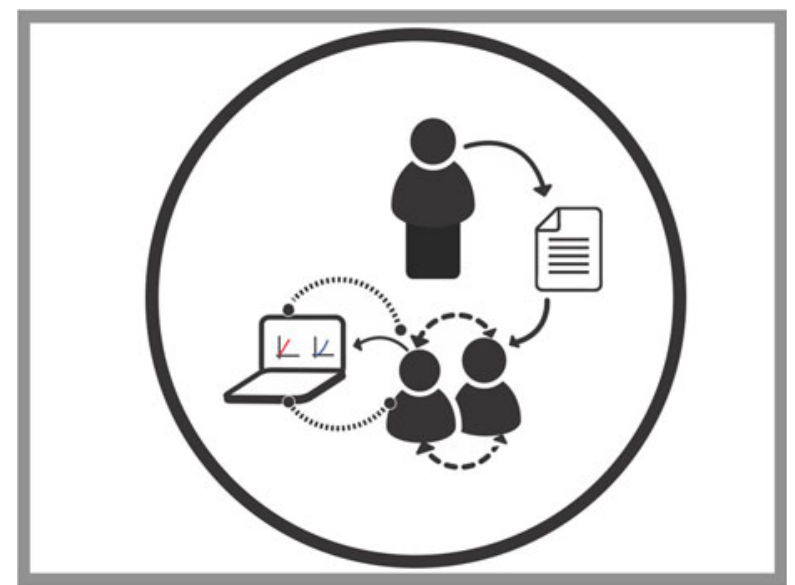

Fig. 24 Representation of a dialogical ecosystem, gathering teacher, students, meaning, mathematical language and digital technology within the classroom 
co-action that takes place through technology. This co-action embodies mathematical knowledge as invariants - those features that do not change with the change due to the intentional dragging. The repertoire of mathematical language is there for students to make a connection with real-world meaning when talking about mathematics knowledge. This is a key element of a dialogic ecosystem: it affords the appropriation of disciplinary knowledge through talk-in-interaction, and it is possible because meaning associated to mathematical knowledge promotes the dialogue.

In this way, we are promoting a mathematical culture, inserting real-world meaning to the learning process. Dialogic ecosystems are committed to inserting a disciplinary conversation into the current classroom culture, and this is possible today because of the evolution of dynamic digital technologies. A dialogic education for mathematics learning necessarily includes the recovery of the meaning of mathematical notions and procedures.

\section{References}

Alanís, J., \& Salinas, P. (2010). Cálculo de una variable: Acercamientos newtoniano y leibniziano integrados didácticamente. El Cálculo y su Enseñanza, 2, 1-14.

Arzarello, F., Olivero, F., Paola, D., \& Robutti, O. (2002). A cognitive analysis of dragging practices in Cabri environments. ZDM: The International Journal on Mathematics Education, 34(3), 66-72.

Bakhtin, M. (1982). The dialogic imagination. Austin, TX: University of Texas Press.

Bakhtin, M. (1986). Speech genres and other late essays. Austin, TX: University of Texas Press.

Burke, J., Hegedus, S., \& Robidoux, R. (2013). Reflections on significant developments in designing SimCalc software. In S. Hegedus \& J. Roschelle (Eds.), The SimCalc vision and contributions (pp. 65-83). Dordrecht, NL: Springer.

Fernández-Cárdenas, J. (2015). Dialogism: sequentiality, positioning, plurality and historicity in the analysis of educational practice. Sinéctica, 43, 1-20.

Goodwin, C. (2007). Participation, stance and affect in the organization of activities. Discourse \& Society, $18(1), 53-73$.

Hegedus, S., \& Moreno-Armella, L. (2010). Accommodating the instrumental genesis framework within dynamic technological environments. For the Learning of Mathematics, 30(1), 26-31.

Heritage, J. (2001). Goffman, Garfinkel and conversation analysis. In M. Wetherell, S. Taylor, \& S. Yates (Eds.), Discourse theory and practice: a reader (pp. 47-56). London, UK: Sage.

Hong, Y., \& Thomas, M. (2013). Graphical construction of a local perspective. In A. Lindmeier \& A. Heinze (Eds.), Proceedings of the 37th conference of the international group for the psychology of mathematics education (Vol. 3, pp. 81-90). Kiel, DE: PME.

Levinson, S. (1983). Pragmatics. Cambridge, UK: Cambridge University Press.

Mercer, N., \& Howe, C. (2012). Explaining the dialogic processes of teaching and learning: the value and potential of sociocultural theory. Learning, Culture and Social Interaction, 1(1), 12-21.

Moreno-Armella, L., \& Hegedus, S. (2009). Co-action with digital technologies. ZDM - The International Journal on Mathematics Education, 41(4), 505-519.

Moreno-Armella, L., \& Sriraman, B. (2005). The articulation of symbol and mediation in mathematics education. ZDM - The International Journal on Mathematics Education, 37(6), 476-486.

Moreno-Armella, L., \& Sriraman, B. (2010). Symbols and mediation in mathematics education. In B. Sriraman \& L. English (Eds.), Theories of mathematics education: seeking new frontiers (pp. 213232). Berlin, DE: Springer.

Moreno-Armella, L., Hegedus, S., \& Kaput, J. (2008). From static to dynamic mathematics: historical and representational perspectives. Educational Studies in Mathematics, 68(2), 99-111.

Sacks, H., Schegloff, E., \& Jefferson, G. (1974). A simplest systematics for the organization of turn-taking for conversation. Language, 50(4), 696-735.

Salinas, P. (2013). Approaching calculus with SimCalc: linking derivative and antiderivative. In S. Hegedus \& J. Roschelle (Eds.), The SimCalc vision and contributions (pp. 383-399). Dordrecht, NL: Springer. 
Salinas, P., \& Alanís, J. (2009). Hacia un nuevo paradigma en la enseñanza del Cálculo dentro de una institución educativa. Revista Latinoamericana de Investigación En Matemática Educativa, 12(3), 355382.

Salinas, P., \& Quintero, E. (2013). Integrating digital technology for the innovation of calculus curriculum. In proceedings of the ASEE annual conference. Atlanta, GA: American Society for Engineering Education (http://www.asee.org/public/conferences/20/papers/8153/view).

Salinas, P., Alanís, J., \& Pulido, R. (2011). Cálculo de una variable: reconstrucción para el aprendizaje y la enseñanza. Didac, 56-57, 62-69.

Salinas, P., Alanís, J., Pulido, R., Santos, F., Escobedo, J. \& Garza, J. (2012a). Cálculo aplicado: Competencias matemáticas a través de contextos (Tomo I). D.F., MX: Cengage Learning.

Salinas, P., Alanís, J., Pulido, R., Santos, F., Escobedo, J. \& Garza, J. (2012b). Cálculo aplicado: Competencias matemáticas a través de contextos (Tomo II). D.F., MX: Cengage Learning.

Salinas, P., Alanís, J., Pulido, R., Santos, F., Escobedo, J. \& Garza, J. (2013). Cálculo aplicado: Competencias matemáticas a través de contextos (Tomo III). D.F., MX: Cengage Learning.

Salinas, P., Quintero, E., \& González-Mendívil, E. (2014). An environment to promote a visual learning of calculus. In H. Arabnia, A. Bahrami, L. Deligiannidis, \& G. Jandieri (Eds.), Proceedings of the 2014 international conference on frontiers in education: computer science and computer engineering (pp. 425429). Las Vegas, NV: CSREA Press.

Schegloff, E. (1992). Repair after next turn: the last structurally provided defense of intersubjectivity in conversation. American Journal of Sociology, 97(5), 1295-1345.

Schegloff, E., Jefferson, G., \& Sacks, H. (1977). The preference for self-correction in the organization of repair in conversation. Language, 53(2), 361-382.

Sinclair, N., \& Robutti, O. (2014). Teaching practices in digital environments. In S. Lerman (Ed.), Encyclopedia of mathematics education (pp. 598-601). Dordrecht, NL: Springer.

Thompson, P., Byerley, C., \& Hatfield, N. (2013). A conceptual approach to calculus made possible by technology. Computers in the Schools, 30(1-2), 124-147.

Vahey, P., Knudsen, J., Rafanan, K., \& Lara-Meloy, T. (2013). Curricular activity systems supporting the use of dynamic representations to foster students' deep understanding of mathematics. In C. Mouza \& N. Lavigne (Eds.), Emerging technologies for the classroom (pp. 15-30). New York, NY: Springer.

Wegerif, R. (2004). The role of educational software as a support for teaching and learning conversations. Computers \& Education, 43(1-2), 179-191.

Yerushalmy, M., \& Swidan, O. (2012). Signifying the accumulation graph in a dynamic and multirepresentation environment. Educational Studies in Mathematics, 80(3), 287-306. 